\title{
A review of the development of radical photopolymerization initiators used for designing light-curing dental adhesives and resin composites
}

\author{
Kunio IKEMURA ${ }^{1}$ and Takeshi ENDO ${ }^{2}$ \\ ${ }^{1}$ Department of Research and Development, Shofu Inc., 11 Kamitakamatsu-cho, Fukuine, Higashiyama-ku, Kyoto 605-0983, Japan \\ ${ }^{2}$ Molecular Engineering Institute, Kinki University, 11-6 Kayanomori, lizuka, Fukuoka 820-8555, Japan \\ Corresponding author, Kunio IKEMURA; E-mail: k-ikemura@shofu.co.jp
}

\begin{abstract}
This paper reviews our recent studies on radical photopolymerization initiators, which are used in the design of light-curing dental adhesives and resin composites, by collating information of related studies from original scientific papers, reviews, and patent literature. The photopolymerization reactivities of acylphosphine oxide (APO) and bisacylphosphine oxide (BAPO) derivatives, and D,L-camphorquinone (CQ)/tertiary amine were investigated, and no significant differences in degree of conversion (DC) were found between BAPO and CQ/amine system ( $p>0.05)$. In addition, a novel 7,7-dimethyl-2,3-dioxobicyclo[2.2.1] heptane-1-carbonyldiphenyl phosphine oxide (DOHC-DPPO=CQ-APO) was synthesized and its ultraviolet and visible (UV-VIS) spectral behavior was investigated. CQ-APO possessed two maximum absorption wavelengths $(\lambda \max )$ at $350-500 \mathrm{~nm}$ [372 nm (from APO group) and $475 \mathrm{~nm}$ (from CQ moiety)], and CQ-APO-containing resins exhibited good photopolymerization reactivity, excellent color tone, relaxed operation time, and high mechanical strength. It was also found that a newly synthesized, water-soluble photoinitiator (APO-Na) improved adhesion to ground dentin.
\end{abstract}

Keywords: Radical photopolymerization, Photoinitiator, Light-curing dental adhesive

\section{INTRODUCTION}

Photopolymerization science and technology have assumed in recent years an increasing relevance in many applications ${ }^{11}$. In particular, the adverse biological effects of ultraviolet light on vital tissues ${ }^{2)}$ have caused visible light-induced radical polymerization to gain fast acceptance in dentistry.

Since an advanced radical polymerization initiator is an extremely valuable component of light-curing dental adhesives and resin composites, numerous studies have tirelessly explored and sought for more advanced photoinitiators.

By virtue of its outstanding performance for lightcuring resins in dentistry, D,L-camphorquinone (CQ)/ tertiary amine system has hitherto been recognized as a valuable visible light initiator since its invention by Dart and Nemcek ${ }^{3)}$ in 1971. On the initiation mechanism, CQ [wavelength of maximum absorbance $\left.\left(\lambda_{\max }\right): 468 \mathrm{~nm}\right]$ undergoes a so-called hydrogen abstraction type of photoinitiation mechanism in which CQ (a sensitizer) absorbs light to form a photoexcitation complex $\left(\mathrm{CQ}^{*}\right.$-amine exciplex) with a tertiary amine (a hydrogen-donating agent) and subsequently generates amine-derived free radicals ${ }^{4}$.

Acylphosphine oxide (APO) and bisacylphosphine oxide (BAPO) derivatives have received considerable attention because of their inherently interesting photochemistry and photophysics and because they are widely employed as photoinitiators in free radical polymerization processes of photoradiation industries ${ }^{5}$, and they have recently gained new attention in dentistry.

Unlike the CQ system, APO and BAPO do not require tertiary amines. They undergo a so-called $\alpha$ cleavage type of photoinitiation mechanism (Norrish type I system) ${ }^{5,6)}$, in which APO undergoes homolytic $\alpha$ cleavage of the $\mathrm{C}-\mathrm{P}$ bond and generates two free radical species, i.e., $\left[-(\mathrm{O}=) \mathrm{C}^{\cdot}\right]$ and $\left[{ }^{\circ} \mathrm{P}(=\mathrm{O})<\right]$, both capable of initiating polymerization. In terms of curing performance, APO- and BAPO-containing resins cured with a violet light-emitting diode (LED) had approximately the same or higher degree of conversion (DC) when compared against a CQ-containing resin cured with a dental quartz-tungsten-halogen light curing unit ${ }^{7}$. However, APO exhibits poor photoinitiation reactivity with dental blue LED (420-510 nm, $\lambda_{\max }: 455 \mathrm{~nm}$ ) because of its wavelength region.

To date, a photoinitiator which contains both $\alpha$ diketone $[-\mathrm{C}(=\mathrm{O})-\mathrm{C}(=\mathrm{O})-]$ and acylphosphine oxide $[>\mathrm{P}(=\mathrm{O})-\mathrm{C}(=\mathrm{O})-]$ groups bound intramolecularly is unknown. In our recent study ${ }^{8}$, a novel $\mathrm{CQ}$ derivative bearing an APO group, i.e., 7,7-dimethyl-2,3-dioxobicyclo [2.2.1] heptane-1-carbonyl-diphenylphosphine oxide (DOHC-DPPO=CQ-APO) was synthesized. Its spectral behavior and photopolymerization reactivity were assessed to investigate its potential applicability in the design of light-curing resin composites.

With regard to water-soluble initiators, it is known that water-reactive tri- $n$-butylborane (TBB) initiator ${ }^{9,10)}$ enabled hydrophobic methyl methacrylate (MMA) to adhere strongly to moist adherends. To expect the initiation performance like TBB initiator, another noteworthy design consideration is a water-soluble visible light photoinitiator, notably, 2-hydroxy-3(3, 4-dimethyl-9-oxo-9H-thioxanthen-2-yloxy)- $N, N, N$ trimethyl-1-propanaminium chloride (QTX). Effects of 
Table 1 Abbreviations of materials and methods used in this review paper

\begin{abstract}
Materials: $C Q$ : D,L-camphorquinone, $\underline{A P O}$ : acylphosphine oxide, $\underline{B A P O}$ : bisacylphosphine oxide, $\underline{B D P O}$ : benzoyldiphenylphosphine oxide, $\underline{T M M P O}: \quad 2,4,6$-trimethylbenzoylmethoxy-phenylphosphine oxide, $\underline{T M D P O}$ : 2,4,6trimethylbenzoyl diphenylphosphine oxide, $\underline{P B D P O}$ : phthanoyl-bis(diphenylphosphine oxide), $\underline{T F B D P O}$ : tetrafluoroterephthanoyl-bis(didiphenylphosphine oxide), $\underline{D F D P O}$ : 2,6-difluorobenzoyl-diphenylphosphine oxide, $\underline{N D P O}$ (1-naphthoyl) diphenylphosphine oxide, IC 819 (Irgacure 819) [=TMMPO]: bis(2,4,6-trimethybenzoyl)-phenylphosphine oxide, IC 1800 (Irgacure 1800): [A: bis(2,6-dimethoxybenzoyl)-2,4,4-trimethyl-pentylphosphine oxide, B: 1-hydroxycyclohexyl-phenyl-ketone; A:B=1:3], $\underline{B C B P O}$ : bis(2,6-dichlorobenzoyl)-(4-propylphenyl)-phosphine oxide, $\underline{D O H C-D P P O=}$ $\underline{C Q-A P O}$ : 7,7-dimethyl-2,3-dioxobicyclo[2.2.1] heptane-1-carbonyldiphenyl phosphine oxide, $\mathrm{CQ}$-COOH: 7,7-dimethyl-2,3dioxobicyclo[2.2.1]heptane-1-carboxylic acid, $\underline{K A}$ : D, L-ketopionic acid, $\underline{C Q-C O C l}$ : 7,7-dimethyl-2,3-dioxobicyclo[2.2.1] heptane-1-carboxylic acid chloride, $M D P P$ : methoxydiphenylphosphine, $\underline{P P D}$ : 1-phenyl-1,2-propaedione, $B C B P O$ : bis $(2,6$ dichlorobenzoyl)-(4-propylphenyl)phosphine oxide, Tin-Lau: dibutyltin dilaurate, $\underline{U D M A}$ : dimethacryloyloxyethyl-2,2,4trimethylhexamethylene diurethane, TMPT: trimethylolpropane trimethacrylate, $\underline{Q T X}$ : 2-hydroxy-3-(3, 4-dimethyl-9oxo-9H-thioxanthen-2-yloxy)- $N, N, N$-trimethyl-1-propanamin-ium chloride, EDAB: ethyl $p$ - $N, N$-dimethylaminobenzoate, PIDAA: $N$-phenyliminodiacetic acid, DMAEM: $N, N$-dimethylaminoethyl methacrylate, BSNa: benzenesulfinic acid sodium salt, $\underline{B P E}$ : 2,2-bis(4-methavryloyloxypolyethoxyphenyl)propane, $\underline{M T Y A}$ : $N$-methacryloyltyrosine amide, $\underline{10-M D P}$ : 10-methacryloxydecyl dihydrogen phosphate, HEMA: 2-hydroxyethyl methacrylate, GM: glycerol monomethacrylate,

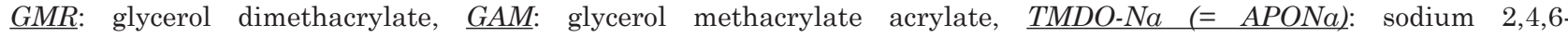
trimethylbenzoyl-phenylphosphine oxide, $\underline{6-M H P A}$ : 6-methacryloyloxyhexyl phosphonoacetate, $\underline{T E G D M A}$ (=3G): triethyleneglycol dimethacrylate, $p$-TSNa: $p$-touluenesulfinic acid sodium salt, $C E$-6: 18-crown-6-ether, 4-META: 4methacryloyloxyethyltrimellitic anhydride, 4-AET: 4-acryloyloxyethyltrimellitic acid, 4-MET: 4-methacryloyloxyethyl trimellitic acid, 4-AETA: 4-acryloyloxyethyltrimellitic anhydride, Bis-GMA: 2,2'-bis[4(2-hydroxy-3-methacryloxypropoxy) phenyl]propane (= bisphenol A diglycidylmethacrylate), $\underline{D M P P}$ : dimethoxy-phenylphosphine, $\underline{V B P O}$ : 4-vinylbenzoyl diphenylphosphine oxide, $\underline{D M V B P O}: \quad 2,6$-dimethyl-4-vinylbenzoyl-diphenylphosphine oxide, $\quad \underline{T M B V P O}$ : 2,4,6trimethylbenzoyl(phenyl-4-vinylphenyl)-phosphine oxide, $\underline{N V P}$ : N-vinylpyrrolidone, $\underline{D A M}$ : dimethylacrylamide, $V B P O: p$ vinylbenzyl-diphenylphosphine oxide, poly(VBPO): polymer of VBPO, poly(VBPO-co-MMA): copolymer of VBPO and methyl methacrylate (MMA), $M C Q$ : D,L-10-methacryloyloxy-camphorquinone, poly(MCQ): polymer of $\mathrm{MCQ}, \underline{p o l y}(M C Q$ $\underline{c o-B M})$ : copolymer of MCQ and BM, poly(DMAEM): polymer of $N, N$-dimethylaminoethyl methacrylate poly(MCQ-co-BM$\underline{c o-D M A E M)}$ : copolymer of MCQ, BM and DMAEM, $\underline{H D A}$ : 1,6-hexanediol-diacrylate, $\underline{B A}$ : $n$-butyl acrylate, $\underline{T B B}$ : tri- $n$ butyl borane, $\underline{T B B O}$ : tri- $n$-butylborane oxide, poly(VBPO): polymer of VBPO, poly(VBPO-co-DAM): copolymer of VBPO and DAM, poly(TMVBPO-co-DAM): copolymer of TMBVPO and DAM
\end{abstract}

Methods: $\underline{U V-V I S}$ spectra: ultraviolet and visible spectra, $\underline{D C}$ : degree of conversion, $\underline{\lambda_{\max }}$ : wavelength of maximum absorption, $\underline{L E D}$ : light-emitting diode, $\underline{Q T H}$ : quartz-tungsten halogen lamp, $\underline{E S R}$ : electron spin resonance, $\underline{F T-I R}$ : Fourier transform infrared spectra, ${ }^{1} \underline{H}$ NMR: proton nuclear magnetic resonance, ${ }^{1} \underline{H}-N M R$-CIDNP: ${ }^{1} \mathrm{H}$ NMR-chemically induced dynamic nuclear polarization, $\underline{D S C}$ : differential scanning calorimeter, $\underline{L B}$ films: Langmuir-Blodgett films

water-soluble QTX in self-etching all-in-one adhesives on adhesion to polished dentin have been well investigated by Hayakawa et al. ${ }^{11-16)}$ and Kikutake et $a l{ }^{17,18)}$ and shown to be effective in improving the bond strength of composite resins to tooth substrates. In light of these encouraging results, a single-step hydrophobic adhesive which contained a water-soluble photoinitiator -sodium acylphosphine oxide (APONa) - and crown ether was synthesized, and its effect on adhesion to ground teeth was investigated ${ }^{19)}$.

Further, to design a single-step self-etching adhesive, our recent study ${ }^{20}$ was carried out to investigate the effect of a single-step, self-etching, HEMA-free adhesive - which contained a phosphonic acid monomer and a carboxylic acid monomer- on adhesion without the use of water-soluble photoinitiators.

The final chapter in this paper will discuss photoinitiating monomers and polymeric photoinitiators because they are now an attractive target for designing of biological injectable prepolymers, which are now widely used for biomedical applications such as bone cements and tooth filling materials.

Although number of light-curing dental adhesives and resin composites are already commercially utilized, little review of the development of photoinitiators has been reported. Against this background of scarcity in published literature, our recent review paper ${ }^{21)}$ discussed the effect of chemically-cured radical polymerization initiators on adhesion.

As for the present review paper, its focus is on our series of research and development studies on radical photopolymerization initiators which can potentially be used in the design of light-curing adhesives and resin composites. The aims of this review paper, therefore, were to discuss the following aspects:

1. Photochemistry of radical photopolymerization and photoinitiators in dentistry;

2. UV-VIS spectra, photopolymerization reactivities, and degrees of conversion of photoinitiators;

3. Effects of photoinitiators with light-curing units on photopolymerization;

4. Development of a novel camphorquinone derivative bearing an acylphosphine oxide group (CQ-APO);

5. Effect of water-soluble photoinitiator, QTX, in self-etching all-in-one adhesive on adhesion;

6. Effect of water-soluble photoinitiator, APO-Na, 
with crown ether on adhesion;

7. Development of a new HEMA-free, self-etching adhesive without use of water-soluble photoinitiators;

8. Photoinitiating monomers and polymeric photoinitiators for biomedical applications.

Numerous materials and methods were used in this paper, and their abbreviations with full names are listed in Table 1.

\section{PHOTOCHEMISTRY OF RADICAL PHOTOPOLYMERIZATION AND PHOTOINITIATORS IN DENTISTRY}

Rapid progress of visible light-induced polymerization technology in dentistry

The photopolymerization technology is based on the use of photoreactive systems (which contain double bonds) suited to absorb a light irradiation of the appropriate wavelength and to produce primary radical species able to convert a multifunctional monomer into a crosslinked network ${ }^{1)}$. The success of this technology hinges on the availability and action of appropriate photoinitiators, because the first step in the photopolymerization process is photoinitiation.

As for the photoinitiators, their success factors are linked to high absorptivity of the photoinitiator in the spectral region corresponding to the irradiating lamp emission, high efficacy in terms of both quantum yield for radical formation and high reactivity of the monomer formulation, good solubility in the curable medium, low odor and toxicity, and good storage stability $^{1)}$.

Considering the adverse health effects of ultraviolet light for oral soft tissues ${ }^{2}$, visible-light induced radical polymerization has fast acceptance of photopolymerization technology in dentistry, and visible-light curing resin system has been rapidly progressing over the past three decades in dentistry rather than other radiation curing industry.

Moreover, compared to chemically cured and ultraviolet curing systems, the outstanding advantages of visible-light curing technology are its one-component nature, consistent handling characteristics, superior physical properties without the problem of microporosity and small voids, and improved adhesion. Coupled with the fast acceptance of photopolymerization technology in dentistry, visible light-induced radical polymerization has since become the widely applied process for bonding resins, resin composites, and composites for crown and bridge applications.

Invention of $C Q /$ tertiary amine photoinitiator system In 1971, Dart and Nemcek ${ }^{3)}$ (Imperial Chemical Industries Co. Ltd., UK) invented a visible light photoinitiator, $\alpha$-diketone/tertiary amine, i.e., $\mathrm{D}, \mathrm{L}$ camphorquinone (CQ)/N,N-dimethylaminoethyl methacrylate (DMAMA). This invention opened a new vista in the field of light-curing dental resins. By virtue of its outstanding performance for light-curing dental resin composites and bonding resins, CQ/tertiary amine system has hitherto been recognized as an extremely valuable visible light initiator since its invention.

Another advantage of the $\alpha$-diketone/tertiary amine system lies in its one-component packaging. This is because both $\alpha$-diketone and tertiary amine can coexist in the dark, thereby enabling the formulations of composite pastes or bonding resins to be packaged as a single component in black or light-shielding containers.

Since $\alpha$-diketon/tertiary amine system can coexist in dark, this photoinitiator system, differ from benzoyl peroxide (BPO)/aromatic tertiary amine, enables the formulation of composite pastes or bonding resins to a single pack component in black or light-shielding container.

\section{Hydrogen abstraction initiating mechanism of CQ/} amine photoinitiators

$\mathrm{CQ}$ displays its photoinitiating activity in combination with synergistic tertiary amines, hence the CQ/tertiary amine system is a so-called hydrogen abstraction (electron donating) type of photoinitiator. The underlying photoinitiation mechanism is that $\mathrm{CQ}$ [wavelength of maximum absorbance $\left(\lambda_{\max }\right): 473 \mathrm{~nm}$ ] absorbs visible rays to form an excited state $\left(\mathrm{CQ}^{*}\right)$, and $\alpha$-hydrogen of tertiary amine (reducing agent=hydrogendonating agent) donates $\mathrm{CQ}^{*}$ to form $\mathrm{CQ}$-amine photoexcitation complex (=exciplex). After the complex decomposes to CQ-ketyl radicals (=hydryl $\cdot \mathrm{C}-\mathrm{OH}$ radicals) and aminyl $\mathrm{C}-\mathrm{N}$ radicals ${ }^{1,4)}$ (Fig. 1), the latter become the actual free radical species which initiate the crosslinking process whereas the former are mainly active in radical-radical recombination reactions ${ }^{1}$.

Although CQ/tertiary amine system offers many advantages, it must be pointed out that the tertiary

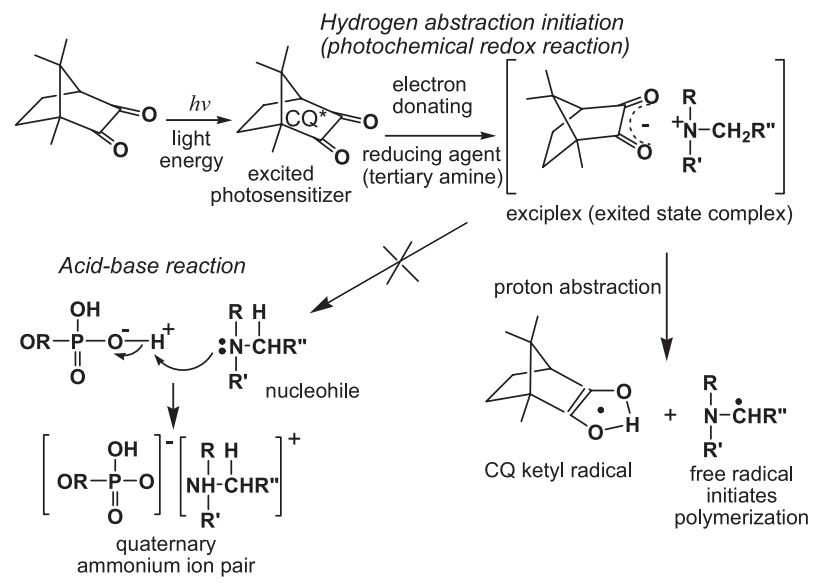

Fig. 1 Hydrogen abstraction photoinitiating mechanism of DL-camphorquinone (CQ) with tertiary amine. Tertiary amine ( $\pi$ acceptor) can react with an acidic group i.e., phosphate or carboxyl group $(\pi$ donor), in adhesion-promoting monomers to form undesirable quaternary ammonium salt ${ }^{4)}$. 

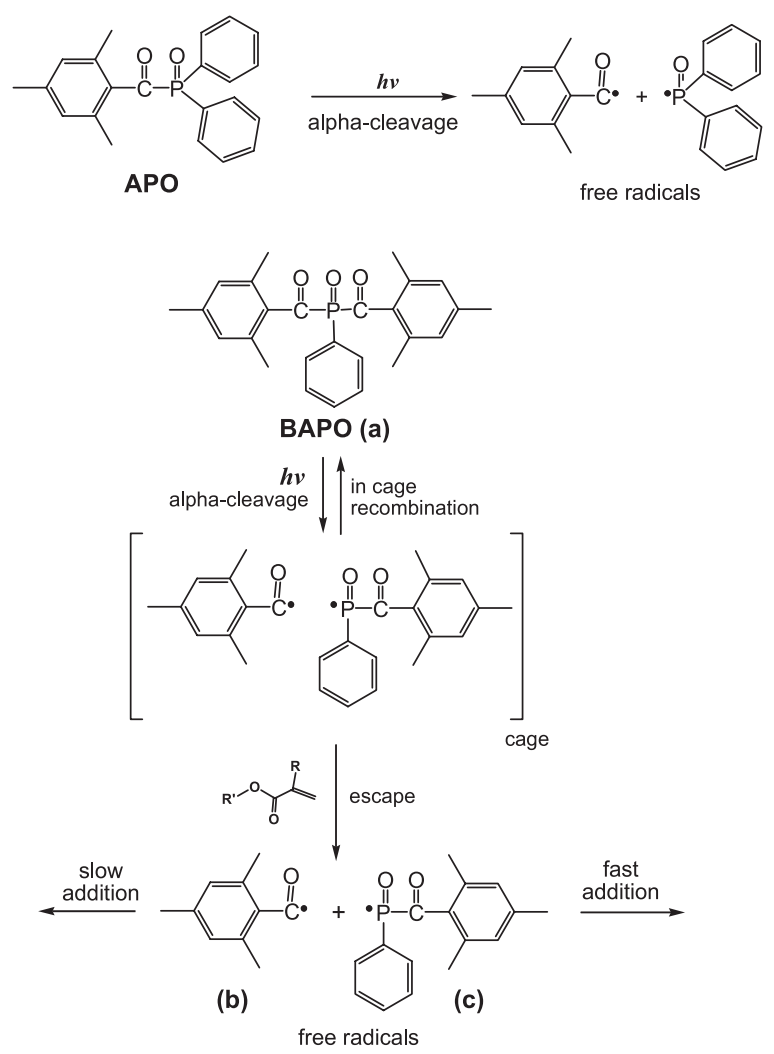

Fig. $2 \quad \alpha$-cleavage photoinitiation mechanism of acylphosphine oxide (APO) and bisacylphosphine oxide $(\mathrm{BAPO})^{5}$. BAPO (a) undergoes initial $\alpha$ cleavage from the triplet-excited state to yield radicals (b) and (c). Radical (c) is 2-6 times more reactive than $(b)^{5)}$.

amine ( $\pi$ acceptor) can react with an acidic group, i.e., phosphoric acid, phosphonic acid, or carboxylic acid group ( $\pi$ donor) in adhesive monomers to form chargetransfer complexes (CT complexes) ${ }^{22,23)}$ or undesirable quaternary ammonium salt ${ }^{4}$ (Fig. 1). It was reported that the formation of quaternary ammonium salt resulted in increased time delay before light activation of the resin composite, hence ultimately resulting in degraded bond strength to dentin ${ }^{4}$.

\section{a-cleavage photoinitiation mechanism of APO and} $B A P O$

During the past several years, most efforts aimed at developing new photoinitiators have focused on developing new compounds that possess higher photoinitiation reactivity. To this end, acylphosphine oxide (APO) was invented by BASF Aktiengesellschaft, Germany and bisacylphosphine oxide (BAPO) by Ciba Specialty Chemicals Corp., NY, USA, and they were launched as commercial products.

Unlike CQ/amine photoinitiator system, APO and BAPO do not require tertiary amines. These photoinitiators undergo an $\alpha$-cleavage type of photoinitiation mechanism (Norrish type I system $)^{5,6}$, where 2,4,6-trimethylbenzoyl-diphenylphosphine oxide
(TMDPO) (=APO) undergoes rapid $\alpha$-cleavage from a triplet excited state to yield the 2,4,6-trimethylbenzoyl radical $\left(\mathrm{CH}_{3}\right)_{3} \mathrm{Ph}-\mathrm{C}^{\cdot}(=\mathrm{O})$ and diphenylphosphinoyl radical $(\mathrm{Ph})_{2} \mathrm{P}^{\cdot}(=\mathrm{O})^{5,6,24-26)}$. Both radicals are capable of initiating polymerization, but with different rate constants $^{5,6)}$ (Fig. 2).

In an investigation by Sumiyoshi et al. ${ }^{6)}$ to elucidate the kinetics and mechanism of the photolysis of TMDPO in the presence of radical polymerizable monomers capable of undergoing $\alpha$-cleavage-initiated photopolymerization, rather high rate constants (in l $\mathrm{mol}^{-1} \mathrm{~s}^{-1}$ ) were found for the reactions of radicals with polymerizable olefinic monomers: $6.0 \times 10^{7}$ (methyl methacrylate), $6.0 \times 10^{7}$ (styrene). The quantum yield (ø) of radical formulation is ø $(-\mathrm{TMDPO})=0.5$ to $0.7^{6)}$.

In another investigation by Kolczak et al. ${ }^{25)}$, the reaction mechanisms of monoacyl- and bisacylphosphine oxide photoinitiators were studied using ${ }^{31} \mathrm{P}-,{ }^{13} \mathrm{C}$ - and ${ }^{1} \mathrm{H}$-NMR-CIDNP (chemically induced dynamic nuclear polarization) and electron spin resonance (ESR) spectroscopies. On the fate of the primary radicals, it was unambiguously shown by trapping experiments that BAPO photoinitiators generated four radicals in a stepwise process from a single precursor - a photochemical mechanism similar to that of APO photoinitiators ${ }^{25)}$. In other words, active photopolymerization could be performed. For example, bis(2,4,6-trimethylbenzoyl)-phenylphosphine oxide $\left.(\mathrm{BTPPO})^{6}\right)(=\mathrm{BAPO})$ (a) underwent initial $\alpha$-cleavage from the triplet excited state to form radicals $\left(\mathrm{CH}_{3}\right)_{3} \mathrm{Ph}$ $\mathrm{C}^{\cdot}(=\mathrm{O})(\mathrm{b})$ and $\left(\mathrm{CH}_{3}\right)_{3} \mathrm{PhC}(=\mathrm{O})-(\mathrm{Ph}) \mathrm{P}^{\cdot}(=\mathrm{O})(\mathrm{c})$, as shown in Fig. 2. It was reported that (c) was $2-6$ times more reactive than $(b)^{5)}$.

\section{UV-VIS SPECTRA, PHOTOPOLYMERIZATION REACTIVITIES, AND DEGREES OF CONVERSION OF PHOTOINITIATORS}

$U V$-VIS spectra and photopolymerization times of APO and $B A P O$ derivatives

The ultraviolet and visible (UV-VIS) spectra and photopolymerization reactivities of APO and BAPO derivatives were investigated in our previous $\mathrm{study}^{27)}$. Figure 3 shows the UV-VIS spectra of 10 kinds of APO and BAPO derivatives as well as their corresponding structural formulas. Their full names are listed in Table 1.

According to their UV-VIS spectra, they possessed $\lambda_{\max }$ ranging between 365 and $416 \mathrm{~nm}$. The $\lambda_{\max }$ of BDPO, NDPO, and IC 819 (=BAPO) were greater than $400 \mathrm{~nm}$. As for the synthesized APO derivatives, there were slight differences in $\lambda_{\max }$ from the ultraviolet region to the visible light region although their structural formulas showed apparent differences. This could be caused by the $[>\mathrm{P}(=\mathrm{O})-\mathrm{C}(=\mathrm{O})-]$ moiety.

Figure 4 shows the method of measuring photopolymerization times using a differential scanning calorimeter (photo-DSC). Results showed that IC 819 (=BAPO), TMDPO (Lab), IC 1800, TMDPO (Com), and TMMPO demonstrated high photoinitiation reactivity 


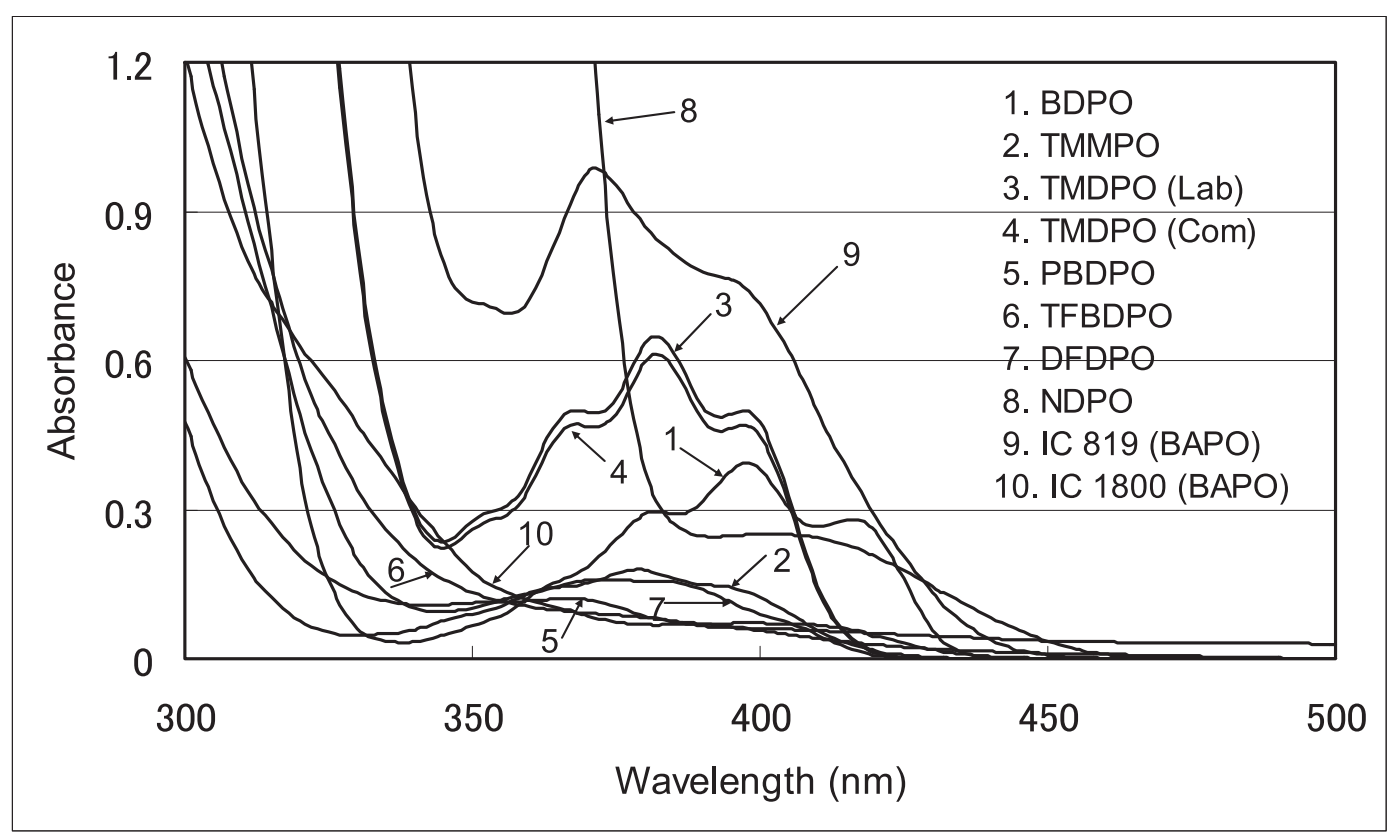

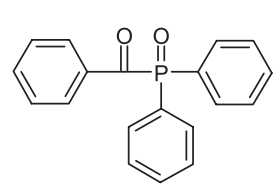

1. BDPO<smiles>COP(=O)(C(=O)c1c(C)cc(C)cc1C)c1ccccc1</smiles>

2. TMMPO<smiles>Cc1cc(C)c(C(=O)P(=O)(c2ccccc2)c2ccccc2)c(C)c1</smiles>

3. TMDPO (Lab)<smiles>Cc1cc(C)c(C(=O)P(=O)(c2ccccc2)c2ccccc2)c(C)c1</smiles>

4. TMDPO (Com)<smiles>O=C(C(=O)P(=O)(c1ccccc1)c1ccccc1)c1ccc(C(=O)P(=O)(c2ccccc2)c2ccccc2)cc1</smiles>

5. PBDPO<smiles>O=C(c1c(F)c(F)c(C(=O)P(=O)(c2ccccc2)c2ccccc2)c(F)c1F)P(=O)(c1ccccc1)c1ccccc1</smiles>

6. TFBDPO<smiles>O=C(c1c(F)cccc1F)P(=O)(c1ccccc1)c1ccccc1</smiles>

7. DFDPO<smiles>O=C(c1cccc2ccccc12)P(=O)(c1ccccc1)c1ccccc1</smiles>

8. NDPO<smiles>Cc1cc(C)c2c(c1)-c1cc(C)cc(C)c1-c1cccc3c1P(=O)(C2=O)C(=O)c1c(C)cc(C)cc1-3</smiles>

9. IC 819 (BAPO)

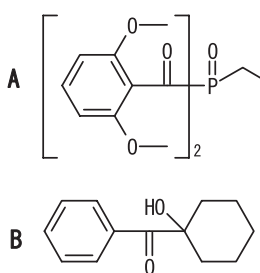

$A: B=1: 3$

10. IC 1800 (BAPO)

Fig. 3 The UV-VIS spectra of 10 kinds of APO and BAPO derivatives and their structural formulas. BDPO, TMMPO, TMDPO (Lab), PBDPO, TFBDPO, DFDPO and NDPO were synthesized in our previous study ${ }^{27}$, whereas TMDPO (Com), IC 819, and IC 1800 were commercial reagents. Full names of all the photoinitiators are given in Table 1.

(Table 2). In sharp contrast, NDPO exhibited a long photopolymerization time (70.0 sec) with a broad photoDSC peak — which might be caused by the naphthoyl group in the structure, while $\lambda_{\max }(401 \mathrm{~nm})$ was slightly shifted to the visible light region ${ }^{27)}$.

Degree of conversion achieved with APO, BAPO and $C Q /$ tertiary amine

Degree of conversion (DC), which can be defined as the degree (\%) of conversion of polymerizable monomers to polymers, is a very important parameter used to measure polymerization performance. In the case of photopolymerization, DC is correlated with the physical properties of light-curing resins and is also influenced by multiple factors: the photopolymerization activity of photoinitiators and photosensitive resin monomers, and the wavelength and intensity of irradiation lamps. For these reasons, thorough and broad-ranging investigations on DC had to be carried out using several photoinitiators. 

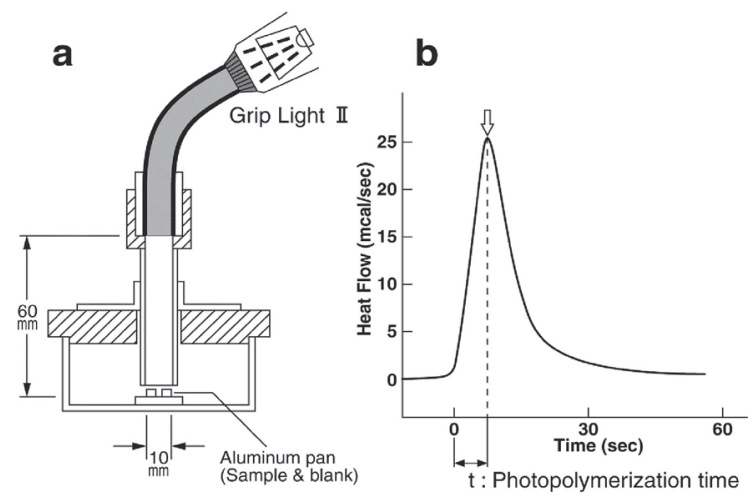

Fig. 4 Measurement of photopolymerization time (sec) with a differential scanning calorimeter (photoDSC) ${ }^{27)}$.<smiles>CC1C(=O)C(=O)C2CCC1(C)C2(C)C</smiles>

$\mathrm{CQ}$<smiles>Cc1cc(C)c(C(=O)C(=O)P(=O)(c2ccccc2)c2ccccc2)c(C)c1</smiles>

TMDPO (APO)<smiles>CC(=O)C(=O)c1ccccc1</smiles>

PPD

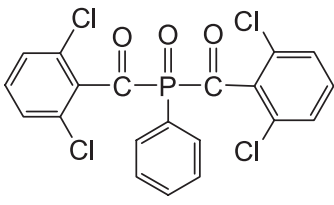

BCBPO (BAPO)

Fig. 5 Structural formulas of photoinitiators investigated by Arikawa et al. ${ }^{7}$. Full names of $\mathrm{CQ}, \mathrm{PPD}$, TMDAPO, and BCBPO are given in Table 1.

Table 2 UV-VIS spectra, physical appearance, melting points, and photopolymerization times of APO and BAPO derivatives investigated ${ }^{27)}$

\begin{tabular}{lllllc}
\hline Photoinitiator & Classified as & \multicolumn{1}{c}{ Appearance } & \multicolumn{1}{c}{$\begin{array}{c}\text { Melting point } \\
(\mathrm{K})\end{array}$} & $\begin{array}{c}\text { UV-VIS spectra } \\
\left(\lambda_{\max }, \mathrm{nm}\right)\end{array}$ & $\begin{array}{c}\text { Photo-time } \\
(\mathrm{sec})\end{array}$ \\
\hline BDPO & APO & light yellow oily product & - & $382,398,416$ & $9.9(0.5)^{\mathrm{a}}$ \\
TMMPO & APO & light yellow oily product & - & 373 & $7.5(0.4)^{\mathrm{b}}$ \\
TMDPO (Lab) & APO & white needle crystals & $359-361$ & $368,382,397$ & $6.7(0.5)^{\mathrm{b}, \mathrm{d}}$ \\
TMDPO (Com) & APO & white powder & $361-365$ & $368,382,397$ & $7.5(0.8)^{\mathrm{b}}$ \\
PBDPO & APO & light yellow powder & $473-478$ & 368 & inactivated \\
TFBDPO & APO & light yellow powder & - & 380 & inactivated \\
DFDPO & APO & light brown oily product & - & $365,379,391$ & $9.3(0.5)^{\mathrm{a}}$ \\
NDPO & APO & light yellow powder & $436-439$ & 401 & $70.0(5.5)^{\mathrm{c}}$ \\
IC 819 & BAPO & light yellow powder & - & 371,400 & $5.9(0.3)^{\mathrm{d}}$ \\
IC 1800 & BAPO & light yellow powder & - & 398 & $6.8(0.5)^{\mathrm{b}, \mathrm{d}}$ \\
\hline
\end{tabular}

Unfilled resins used: Bis-GMA/3G (60/40, wt\%). Concentration of photoinitiators: 1.0 wt\%. BDPO, TMMPO, TMDPO (Lab), PBDPO, TFBDPO, DFDPO, and NDPO were synthesized; TMDPO (Com), IC 819 and IC 1800 were commercial reagents. Photo-time: photopolymerization time (sec); $n=5$. Light intensity used for photo-DSC was $100 \mathrm{mWcm}^{-1}$. Groups from the same column that are identified with the same superscript letter are not significantly different $(p>0.05)$ Full names of photoinitiators are given in Table 1.

Table 3 Degrees of conversion (\%) of unfilled resins initiated by BAPO (IC1800) and CQ/EDAB initiator systems ${ }^{27)}$

\begin{tabular}{lcccc}
\hline \multirow{2}{*}{$\begin{array}{l}\text { Unfilled resins } \\
\text { (a)/(b) }\end{array}$} & \multicolumn{2}{c}{ BAPO (IC 1800) initiator } & \multicolumn{2}{c}{ CQ/EDAB system } \\
\cline { 2 - 5 } $100 / 0$ & Group A & Group B & Group A' & Group B' \\
$80 / 20$ & $47.8(6.7)^{\alpha}$ & $47.4(5.2)^{\alpha}$ & $45.6(2.8)^{\alpha}$ & $46.1(3.1)^{\alpha}$ \\
$60 / 40$ & $46.9(6.5)^{\alpha}$ & $49.2(1.0)^{\alpha}$ & $45.4(2.3)^{\alpha}$ & $46.2(4.4)^{\alpha}$ \\
$40 / 60$ & $46.2(4.6)^{\alpha}$ & $51.2(4.3)^{\alpha}$ & $44.9(4.2)^{\alpha}$ & $47.5(3.7)^{\alpha}$ \\
$20 / 80$ & $46.6(5.5)^{\alpha}$ & $45.2(4.1)^{\alpha}$ & $42.4(4.4)^{\alpha}$ & $45.7(3.6)^{\alpha}$ \\
$0 / 100$ & $44.2(6.5)^{\alpha}$ & $44.4(2.4)^{\alpha}$ & $43.3(5.7)^{\alpha}$ & $43.0(4.8)^{\alpha}$ \\
$100 \%$ OH-monomer & did not test & did not test & did not test & did not test \\
\hline
\end{tabular}

$n=5$. Concentration of photoinitiators: BAPO (2.0 wt\%), CQ/EDAB (0.5/1.0, wt\%). OH-monomer: Group A and A': HEMA, Group B and B': GM. Group A and A': (a) Bis-GMA/3G (60/40, wt\%)/(b) 6-MHPA/HEMA (50/50, wt\%), Group B and B': (a) Bis-GMA/3G (60/40, wt\%)/(b) 6-MHPA/GM (50/50, wt\%). Groups from the same column that are identified with the same superscript letter are not significantly different $(p>0.05)$. Full names of CQ, HEMA, GM, Bis-GMA, 3G, 6-MHPA, BAPO, IC 1800, CQ, and EDAB are given in Table 1. 
In our recent study ${ }^{27)}$ on the photopolymerization of APO-, BAPO- and CQ/tertiary amine-containing unfilled resins, DC (\%) —as the degree of double bond conversion - was determined using Fourier transform infrared (FTIR) spectroscopy. As for the method reported in previous studies ${ }^{28,29)}$, DC was obtained based on the subtraction of FTIR spectra measured before and after 30 seconds of light irradiation, using the residual percentage of polymerizable aliphatic double bond from $100 \%$.

Table 3 presents the results of DC obtained. For the BAPO system, the DC values were 44.2(6.5)-51.2(4.3)\%; for the CQ/ethyl $p-N, N$ dimethylaminobenzoate (EDAB) system, the DC values were $42.4(4.4)-47.5(3.7) \%$. Statistical analysis showed that there were no significant differences between the BAPO and CQ/EDAB systems among the different concentrations of 6-methacryloyloxyhexyl phosphonoacetate (6-MHPA)/HEMA and 6-MHPA/ glycerol monomethacrylate (GM) in 2,2'-bis[4(2hydroxy-3-methacryloxypropoxy)phenyl]propane (BisGMA)/triethyleneglycol dimethacrylate [TEGDMA $(=3 \mathrm{G})]$ resins $(p>0.05)$.

\section{Degree of conversion achieved with CQ/PPD system} Park et al. ${ }^{29)}$ examined the synergistic effect of combining CQ with 1-phenyl-1,2-propanedione (PPD) (Fig. 5) as a new photoinitiator. PPD alone induced a DC that was not significantly different from that of $\mathrm{CQ}$ alone. When used in combination, CQ+PPD produced a DC which generally exceeded that produced by the same concentration of either used alone. Further, DC was increased by PPD but depressed by CQ, which was evidence that different mechanisms were involved. It should also be mentioned that PPD $\left(\lambda_{\max }: 410 \mathrm{~nm}\right)$ and CQ $\left(\lambda_{\max }: 473 \mathrm{~nm}\right)$ had different wavelength absorption ranges.

After 20 seconds of visible light irradiation with CQ/PPD system, DC obtained was $53 \%{ }^{29)}$. This was almost comparable with the DC values achieved with $\mathrm{BAPO}$ or $\mathrm{CQ} / \mathrm{EDAB}$ in our study ${ }^{27)}$, set against the backdrop that different photoinitiators and compositions were employed in both studies ${ }^{27,29)}$.

\section{EFFECTS OF PHOTOINITIATORS WITH LIGHT CURING UNITS ON PHOTOPOLYMERIZATION}

DC (\%) produced by four kinds of photoinitiators with four kinds of light curing units

Although APO $\left(\lambda_{\max }: 370 \mathrm{~nm}\right)$ exhibited excellent polymerization reactivity with violet LED lamp irradiation, it provided poor reactivity with dental blue LED lamp $\left(420-510 \mathrm{~nm}, \lambda_{\max }: 455 \mathrm{~nm}\right)$ because of its wavelength.

In a recent study by Arikawa et al. ${ }^{7)}$, the effects of photoinitiators with light curing units on the photopolymerization efficacy of unfilled light-curing resins were investigated. Figure 5 depicts the chemical structures of the four kinds of photoinitiators investigated - namely CQ, PPD, TMDPO, and bis(2,6- dichlorobenzoyl)-(4-propylphenyl)phosphine oxide $(\mathrm{BCBPO}=\mathrm{BAPO})$. Each photoinitiator (1.5 wt\%) was dissolved in a Bis-GMA (50 mol\%) and TEGDMA (50 mol\%) monomer mixture together with ethyl $p$ dimethylamino benzoate (EDAB) $(0.2$ wt\%) as a reducing agent. Prepared unfilled resins were polymerized using a quartz-tungsten-halogen lamp (QTH), xenon plasma arc lamp (PAC), blue LED light curing unit, or a custom-made violet LED light unit.

Results showed that CQ- and TMDPO-containing resin could not be polymerized with the violet LED lamp and blue LED lamp respectively ${ }^{7}$. PPD-, TMDPO-, and BAPO-containing resins (Bis-GMA/ TEGDMA) polymerized with a violet LED light unit had approximately the same or higher degree of DC as compared with CQ-containing resin polymerized with a QTH lamp. On the overall, DC varied according to the combination of photoinitiator and curing light source. The ranges of DC values achieved with each light source were as follows: QTH: $64.8 \%$ (CQ)-75.5\% (TMDPO); PAC: $51.1 \% \quad$ (TMDPO)-58.1\% (BAPO=IC 819); B-LED: 65.4 (PPD)-68.8\% (CQ); V-LED: 61.4\% (PPD)-77.5\% (BAPO).

Photoinitiators activated with dental halogen, blue LED and Xenon lamps

Jandt et $a l .{ }^{30)}$ reported that the depth of cure and compressive strength of dental composites cured with a blue LED lamp were statistically equivalent to those cured with a conventional halogen lamp. This could be attributed to the advantage of a constant power output over the lifetime of the diodes, such that LED lamps are capable of achieving a clinically consistent quality of composite cure.

By means of ESR spectroscopy with a tapping method, Teshima et al. ${ }^{31,32)}$ quantified the primary radicals generated by irradiation by using phenyl-tertbutyl nitrone $(\mathrm{PBN})$ as a spin trapping agent. In their investigations ${ }^{31,32)}$, two types of photoinitiator systems - $\mathrm{CQ} / N, N$-dimethyl- $p$-toluidine (DMPT) and $\mathrm{CQ} / N, N$ dimethylaminoethyl methacrylate (DMAEMA), and three types of light curing units - a blue LED unit [LUX-O-MAX (Panasonic Dental Co., Osaka, Japan)] and two conventional halogen units [Optilux 501 (Demetron/Kerr, Danbury, CT, USA) and XL 3000 (3M ESPE, St. Paul, MN, USA)] were used. Obtained peak wavelengths (nm) of the different light curing units were: LUX-O-MAX at $470 \mathrm{~nm}(430-525 \mathrm{~nm})$, Optilux at $476 \mathrm{~nm}(375-530 \mathrm{~nm})$, and XL3000 at $480 \mathrm{~nm}(400-530$ $\mathrm{nm})$.

Results showed that the CQ/DMPT photoinitiator system generated more radicals than the CQ/DMAEMA system $^{31,32)}$. It was suggested that DMAEMA molecules, which had methacryloyl group, produced DMAEMAdimers or oligomers when radicals existed. On the influence of light curing units, the blue LED light of LUX-O-MAX generated 1.45-1.69 times as many radicals as the visible light of the halogen units. The latter result thus suggested that the LED unit, LUX-OMAX, performed better than the conventional halogen 
<smiles></smiles><smiles></smiles>

Fig. 6 Structural formulas of ketocoumarin compounds $\mathrm{s}^{34)}$. CBEC: 3,3'-carbonylbis(7-diethylaminocoumarin), CBBC: 3,3'-carbonylbis(7-dibutylamino-coumarin).

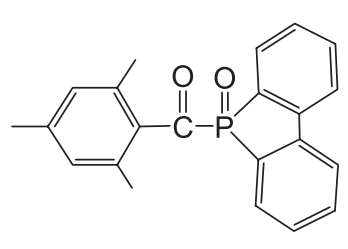

TMBOPF

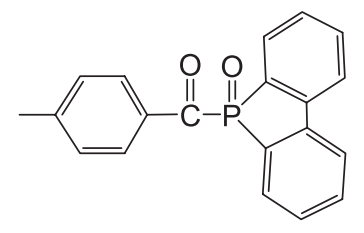

TOPF
Fig. 7 Structural formulas of novel acylphosphine oxide photoinitiators ${ }^{35)}$. TMBOPF: 9-(2,4,6-trimethylbenzoyl)9-oxytho-9-phosphafuluorene, TOPF: 9-(p-toluyl)9-oxytho-9-phosphafuluorene.

light curing units with respect to the generation of primary radicals, i.e., energy efficiency.

Recent patents ${ }^{33,34)}$ claimed that the newly developed photoinitiator systems possessed good photoinitiation activities with dental halogen, blue LED and Xenon lamps. They are physically mixed, visible light curing initiators consisting of $\mathrm{CQ}$, amine, and $\mathrm{APO}^{33}$ or a system comprising APO or BAPO, accelerators, ketocoumarin compounds such as 3,3'carbonylbis(7-diethylaminocoumarin) (CBEC) and 3,3'carbonylbis(7-dibutylamino-coumarin) (CBBC) (Fig. 6), and with/without $\alpha$-diketone ${ }^{34)}$.

Novel acylphosphine oxide photoinitiators: TMBOPF and TOPF

In a recent patent ${ }^{35)}$, novel acylphosphine oxide photoinitiators with a phosphol group were invented. They were namely, 9-(2,4,6-trimethylbenzoyl)-9-oxytho9-phosphafuluorene (TMBOPF) and 9-( $p$-toluyl)-9oxytho-9-phosphafuluorene (TOPF) (Fig. 7). To achieve the TMBOPF structure, the diphenylphosphine oxide moiety $\left[>\mathrm{P}(=\mathrm{O})-(\mathrm{Ph})_{2}\right]$ of APO was displaced with biphenyl phosphine oxide moiety $[>\mathrm{P}(=\mathrm{O})-(\mathrm{Ph}-\mathrm{Ph})]$ to form a five-member ring phosphol moiety.

Unlike conventional APOs, the novel TMBOPF and
TOPF photoinitiators exhibited good photopolymerization reactivity in both visible light and ultraviolet light ranges. In particular, TOPFcontaining Bis-GMA-type resin exhibited good photopolymerization reactivity with a dental visiblelight curing unit, and that its reactivity was comparable with that of BAPO (=IC 819) rather than that of APO.

On color stability, it was disclosed that the degree of yellow (DY) values of thick photo-cured coating films initiated by TMBOPF (DY: 1.9) and TOPF (DY: 2.3) with ultraviolet light irradiation were extremely reduced when compared against those of conventional APO (DY: 4.0) and BAPO (DY: 6.3) ${ }^{35)}$. With sunlight irradiation, the DY obtained with TOPF (DY: 5.7) was lower than that of BAPO (DY: 16.1). On formulation stability, the light-curing resin containing TMBOFP was more stable than that containing TOPF (our unpublished data).

\section{DEVELOPMENT OF A NOVEL CAMPHORQUINONE DERIVATIVE BEARING AN ACYLPHOSPHINE OXIDE GROUP (CQ-APO)}

Rationale behind the design of CQ-APO and its synthesis

Owing to its absorption wavelength, CQ has an inherently large $b$ value in the CIELab color specification system, which is consequently manifested as a yellow tinge. On $\mathrm{CQ}$ derivatives, a Japanese patent $^{36)}$ disclosed that 10 kinds of CQ derivatives, such as 2,3-dioxobicyclo[2.2.1] heptane with adhesive functional groups, possessed $\lambda_{\max }$ ranging between 459 and $472 \mathrm{~nm}$. In other words, they could initiate visible light polymerization like CQ. Although these CQ derivatives were expected to be effective in promoting adhesion, concerns related to color tone remained to be investigated and addressed.

A recent patent ${ }^{33)}$ claimed that a physically mixed, visible light-curing initiator consisting of $\mathrm{APO}, \mathrm{CQ}$, and aliphatic amine exhibited excellent photopolymerization reactivity with both visible light and ultraviolet irradiators. However, this new invention ${ }^{33)}$ did not resolve the key CQ-related problems, namely short operation time and a high $b$ value which was manifested as a tinge of yellow. In light of these unresolved problems, a research study ${ }^{8)}$ was undertaken to develop a new photoinitiator which possessed reduced $b$ value and high photopolymerization reactivity, but lengthened operation time -hence permitting a relaxed mode of therapeutic operation under ambient light.

To date, a photoinitiator having both $\alpha$-diketone [$\mathrm{C}(=\mathrm{O})-\mathrm{C}(=\mathrm{O})-]$ and acylphosphine oxide $[>\mathrm{P}(=\mathrm{O})-\mathrm{C}(=\mathrm{O})-]$ moieties bound intramolecularly is unknown. Our recent study $^{8)}$ thus newly designed and synthesized a novel 7,7-dimethyl-2,3-dioxobicyclo[2.2.1] heptane-1carbonyl-diphenylphosphine oxide (DOHC-DPPO = CQAPO).

Figure 8 is a schematic illustration of the synthesis of CQ-APO, which was synthesized by 


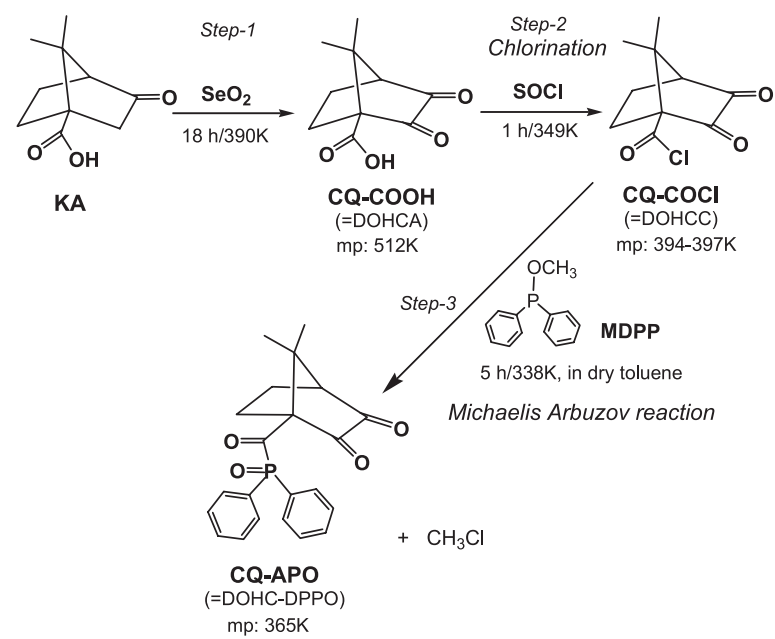

Fig. 8 Schematic illustration of the synthesis of 7,7dimethyl-2,3-dioxobicyclo[2.2.1]heptane-1-carbonyldiphenylphosphine oxide (CQ-APO) ${ }^{8}$. Full names of $\mathrm{KA}, \mathrm{CQ}-\mathrm{COOH}, \mathrm{CQ}-\mathrm{COCl}$, and MDPP are given in Table 1.

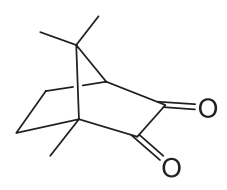

$\mathrm{CQ}$

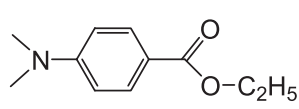

EDAB

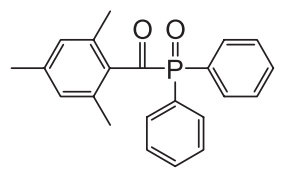

TMDPO

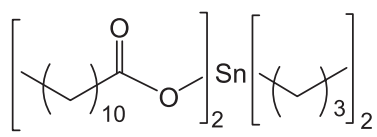

Tin-Lau

Fig. 9 Structural formulas of the photoinitiators or sensitizers and accelerators employed in the synthesis $^{8}$, except CQ-APO. Full names of CQ, TMDPO, EDAB, and Tin-Lau are given in Table 1.

Michaelis-Arbuzov reaction ${ }^{37,38)}$ between methoxydiphenylphosphine (MDPP) $\left[\mathrm{CH}_{3} \mathrm{O}-\mathrm{P}(\mathrm{Ph})_{2}\right]$ and 7,7dimethyl-2,3-dioxobicyclo[2.2.1]-heptane-1-carboxylic acid chloride (DOHCC $=\mathrm{CQ}-\mathrm{COCl}$ ). During this transformation, a tervalent phosphorus [P(III)] (=MDPP) was converted into a pentavalent phosphorus $[\mathrm{P}(\mathrm{V})]$ compound (=CQ-APO). As for CQ-COCl, it was synthesized by the reaction between 7,7-dimethyl-2,3dioxobicyclo[2.2.1]-heptane-1-carboxylic acid $(\mathrm{DOHCA}=\mathrm{CQ}-\mathrm{COOH})$ and thionyl chloride. Figure 9 shows the structural formulas of the photoinitiators or sensitizers and accelerators employed in the synthesis ${ }^{8)}$, except CQ-APO.

UV-VIS spectral behaviors of CQ-APO, CQ, and $T M D P O$

Figures 10-12 show the UV-VIS spectral behaviors of $\mathrm{CQ}-\mathrm{APO}, \mathrm{CQ}$, and TMDPO in dry toluene before and

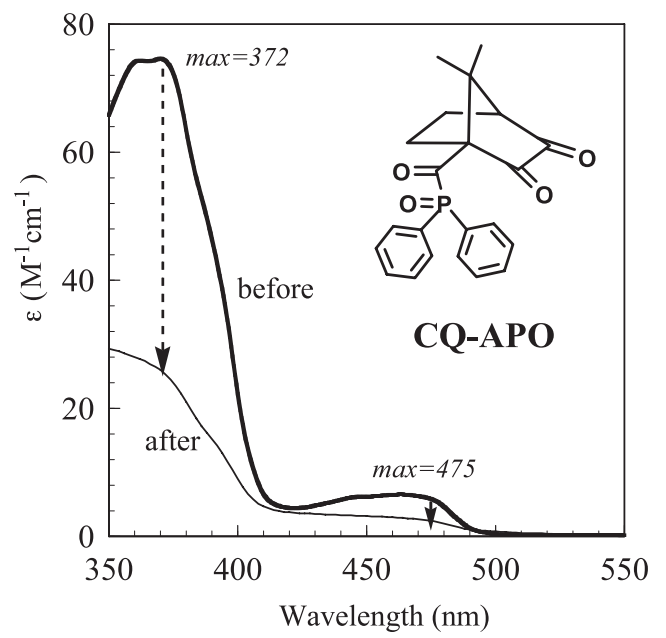

Fig. 10 UV-VIS spectra of CQ-APO in dry toluene before and after light activation with a halogen lamp (light intensity: $650 \mathrm{~mW} / \mathrm{cm}^{2}$, wavelength region: $\left.375-525 \mathrm{~nm}, \lambda_{\max }=500 \mathrm{~nm}\right)^{8}$.

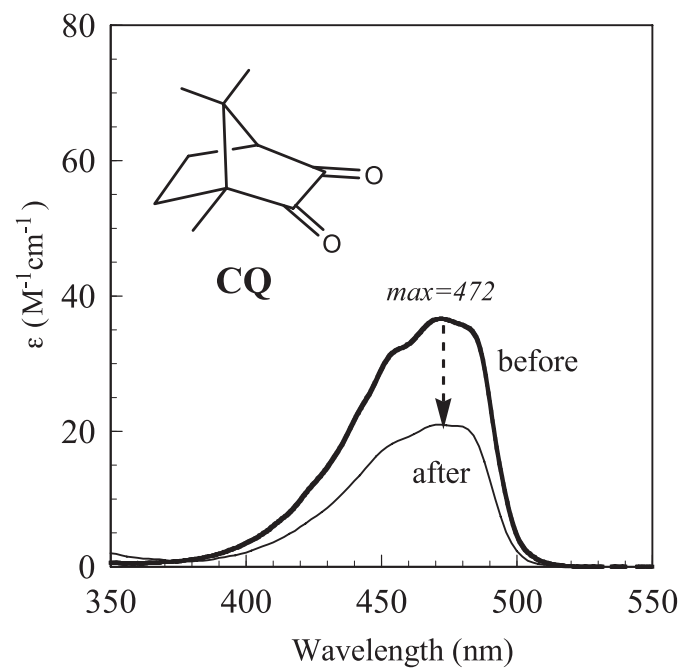

Fig. 11 UV-VIS spectra of CQ in dry toluene before and after light activation with a halogen lamp (light intensity: $650 \mathrm{~mW} / \mathrm{cm}^{2}$, wavelength region: $375-25$ $\left.\mathrm{nm}, \lambda_{\max }=500 \mathrm{~nm}\right)^{8)}$.

after light irradiation with a dental halogen lamp. On the UV-VIS spectrum before light irradiation, $\mathrm{CQ}$ and TMDPO (=APO) showed $\lambda_{\max }=472 \mathrm{~nm}$ and $\lambda_{\max }=382 \mathrm{~nm}$ respectively. It was noteworthy that $\mathrm{CQ}-\mathrm{APO}$ showed continuous wide absorption from near-ultraviolet to visible region at $350-500 \mathrm{~nm}$, whereby $\lambda_{\max }=372 \mathrm{~nm}$ was derived from the acylphosphine oxide group and $\lambda_{\max }=475 \mathrm{~nm}$ was derived from the $\alpha$-diketone group.

On absorption coefficients, TMDPO exhibited a greater value than CQ-APO and which was considerably reduced to $19.0 \mathrm{M}^{-1} \mathrm{~cm}^{-1}(1 / 325)$ after light irradiation. As the latter value was approximately the same as that of CQ-APO, it could be said that the C-P 


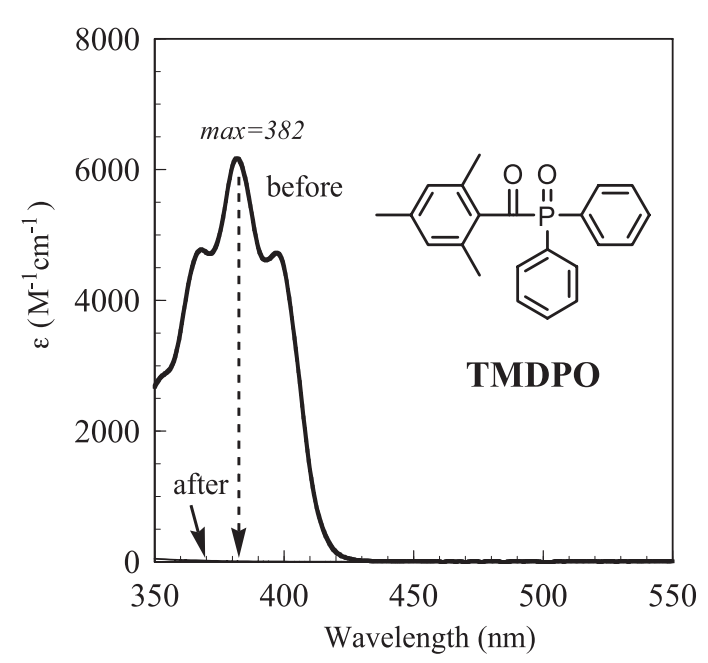

Fig. 12 UV-VIS spectra of TMDPO in dry toluene before and after light activation with a halogen lamp (light intensity: $650 \mathrm{~mW} / \mathrm{cm}^{2}$, wavelength region: $\left.375-525 \mathrm{~nm}, \lambda_{\max }=500 \mathrm{~nm}\right)^{8)}$.

cleavage of $[-\mathrm{C}(=\mathrm{O})-\mathrm{P}(=\mathrm{O})<]$ bond around $380 \mathrm{~nm}$ was generated in both CQ-APO and TMDPO after light irradiation, via $\alpha$-cleavage type of photoinitiation mechanism (Norrish type I system) ${ }^{5,6)}$. Unlike $\alpha$ cleavage type, as for $\mathrm{CQ}$, slow hydrogen abstraction from the toluene solvent did not lead to considerable reduction in absorption coefficient.

Relationship between color tone and spectral absorption It was surprisingly found that CQ-APO - in which CQ and APO groups were bound intramolecularlyexhibited excellent color tone stability with a small $b$ value of 4.0 (Table 4). In contrast, conventional CQ alone and physically mixed CQ/TMDPO exhibited large $b$ values of 84.0 and 87.8 respectively. Therefore, it was apparent that CQ-APO provided considerable improvement in color tone ${ }^{8)}$.

On the relationship between color tone and absorption spectrum, a substance which absorbs light in the visible light region (approx. 400-800 nm) reflects a color whereas a substance which absorbs light in the near-ultraviolet region does not reflect any color. On this premise, CQ appeared yellow whereas TMDPO had a colorless appearance. For CQ-APO, a colorless appearance like that of TMDPO was presented when maximum absorption occurred at $\lambda_{\max }=372 \mathrm{~nm}$ in the near-ultraviolet region. However, for absorption in the visible light region at $\lambda_{\max }=475 \mathrm{~nm}$, a color was reflected.

When the absorption coefficient of CQ-APO was compared with that of $\mathrm{CQ}$ circa $470 \mathrm{~nm}$, the former was about 1/5 that of the latter (Figs. 10 and 11). Owing to this considerable difference in absorption coefficient values, CQ-APO appeared as a very pale yellow crystal as compared to the intense yellow appearance of CQ. Based on these observations, it was

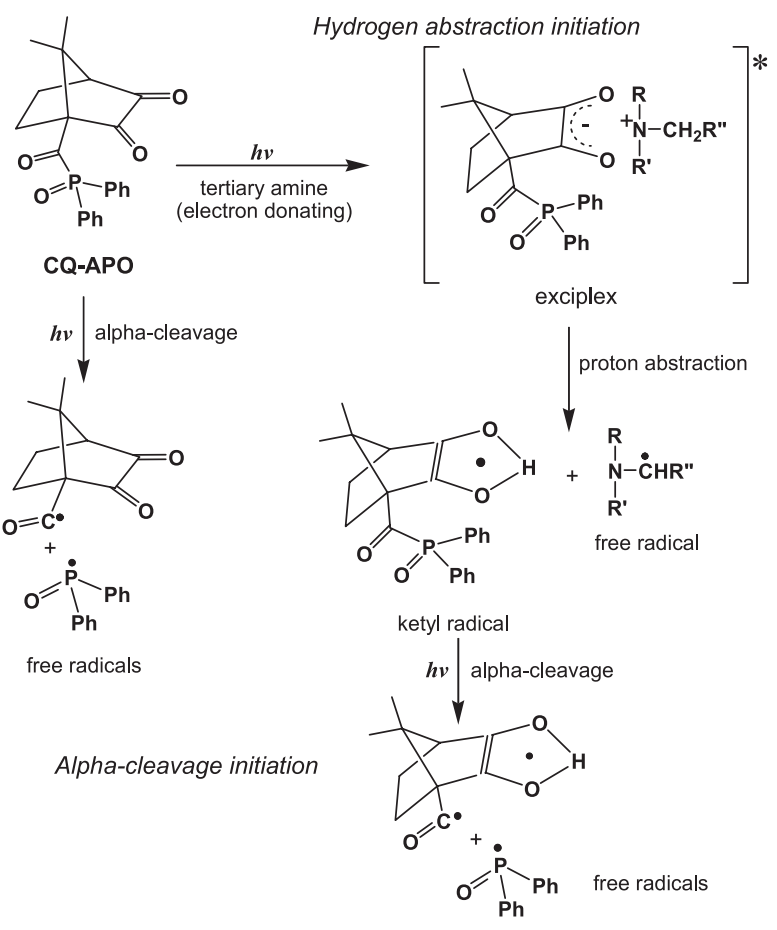

Fig. 13 Schematic illustration of a plausible photoinitiation mechanism of CQ-APO ${ }^{8}$. CQ-APO could undergo both $\alpha$-cleavage initiation to form the $\left[\mathrm{CQ}-(\mathrm{O}=) \mathrm{C}^{\cdot}\right]$ and $\left[{ }^{\cdot} \mathrm{P}(=\mathrm{O})(\mathrm{Ph})_{2}\right]^{5,6)}$ radicals as well as hydrogen abstraction initiation to form CQ-APO ketyl radical and free radicals via the formation of $\mathrm{CQ}^{*}$-APO-amine exciplex. Moreover, the CQ-APO ketyl radical may undergo C-P cleavage to form free radicals ${ }^{8)}$.

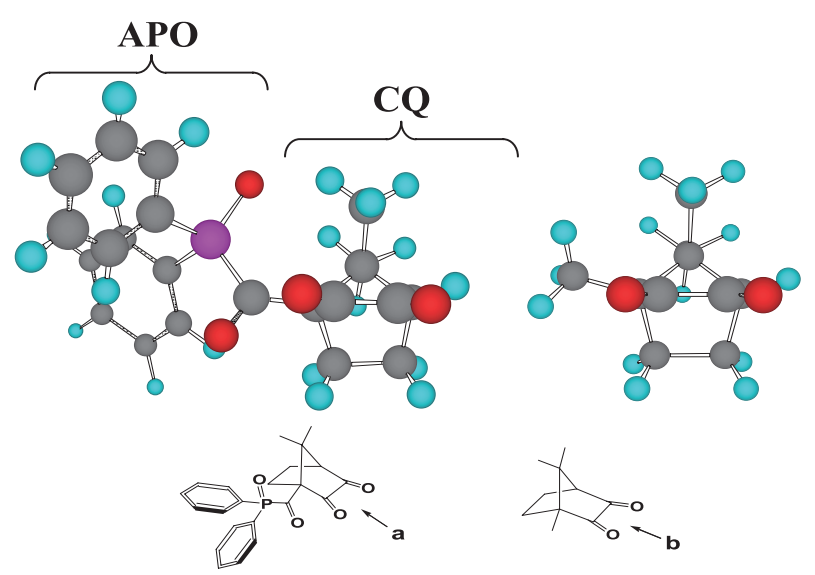

Fig. 14 Stereochemical structures of CQ-APO (left) and $\mathrm{D}, \mathrm{L}$-camphorquinone (right) from the views of $\alpha$ diketone group indicated by arrows a and b by $\alpha$ diketone group ${ }^{8}$. Atoms: blue balls $=\mathrm{H}$, black balls $=\mathrm{C}$, red balls $=\mathrm{O}$, pink balls $=\mathrm{P}$. In a stable structure in potential energy, $\alpha$-diketone group of CQ-APO had a slightly strained structure while that of CQ had a plane structure ${ }^{8)}$. 
presumed that CQ-APO possessed a $b$ color parameter of very small value.

\section{Plausible photoinitiation mechanism of novel $C Q-A P O$} CQ-APO possessed both APO moiety $\left(\lambda_{\max }=372 \mathrm{~nm}\right)$ and $\mathrm{CQ}$ moiety $\left(\lambda_{\max }=475 \mathrm{~nm}\right)$ in its chemical structure. Therefore, it was plausible to suggest that upon light activation, both $\alpha$-cleavage type and hydrogen abstraction type of initiation mechanisms were triggered within CQ-APO.

Figure 13 is a schematic illustration of the plausible photoinitiation mechanism of CQ-APO. It was suggested that CQ-APO underwent rapid $\alpha$ cleavage of the $[-\mathrm{C}(=\mathrm{O})-\mathrm{P}(=\mathrm{O})<]$ bond from a triplet state to afford the [CQ- $(\mathrm{O}=) \mathrm{C} \cdot]$ and diphenylphosphinoyl $\left[\cdot \mathrm{P}(=\mathrm{O})(\mathrm{Ph})_{2}\right] \quad$ radicals $^{5,6)}$, like the photoinitiation mechanism of acylphosphine oxide ${ }^{25,27)}$. In the case of CQ-APO with an electron donating agent such as a tertiary amine, it is also noteworthy that after $\alpha$ diketone $[-\mathrm{C}(=\mathrm{O})-\mathrm{C}(=\mathrm{O})-]$ of $\mathrm{CQ}$-APO absorbed the energy of visible rays to convert into an excited state $\left(\mathrm{CQ}^{*}\right.$-APO), $\alpha$-hydrogen of tertiary amine would donate $\mathrm{CQ}^{*}$-APO to form $\mathrm{CQ}^{*}$-APO-amine exciplex (excited state complex). The latter might be subsequently decomposed to CQ-APO ketyl radical and free radicals derived from tertiary amines, like the photoinitiation mechanism of camphorquinone ${ }^{27)}$. Moreover, unlike an inactivated CQ-ketyl radical, the CQ-APO ketyl radical might undergo C-P cleavage to form free radicals (Fig. 13). To gain more insight into the kinetics of the

Table 4 Compositions and photopolymerization of experimental, visible light-curing, unfilled resins ${ }^{8)}$

\begin{tabular}{|c|c|c|c|c|c|c|c|}
\hline $\begin{array}{l}\text { Ingredients } \\
\text { (parts by weight) }\end{array}$ & UR-1 & UR-2 & UR-3 & UR-4 & UR-5 & UR-6 & UR-7 \\
\hline CQ-APO & 2.0 & - & - & - & 2.0 & - & - \\
\hline $\mathrm{CQ}$ & - & 2.0 & - & 2.0 & - & 2.0 & 2.0 \\
\hline TMDPO & - & - & 1.0 & 1.0 & - & - & 1.0 \\
\hline EDAB & - & - & - & - & 1.0 & 1.0 & 1.0 \\
\hline Bis-GMA & 60.0 & 60.0 & 60.0 & 60.0 & 60.0 & 60.0 & 60.0 \\
\hline TEGDMA & 40.0 & 40.0 & 40.0 & 40.0 & 40.0 & 40.0 & 40.0 \\
\hline Reactivity (Hal/30 sec) & A & $\mathrm{C}$ & A & A & A & A & A \\
\hline Reactivity (LED/10 sec) & $\mathrm{B}$ & $\mathrm{C}$ & $\mathrm{D}$ & $\mathrm{C}$ & A & A & A \\
\hline Photo-time (sec) & NT & NT & NT & NT & $9.6(0.5)^{\mathrm{a}}$ & $10.9(0.7)^{\mathrm{a}}$ & $8.5(0.6)^{\mathrm{a}}$ \\
\hline Operation time (sec) & NT & NT & NT & NT & $50.0(2.5)^{\alpha}$ & $5.0(1.0)^{\beta}$ & $5.0(0.5)^{\beta}$ \\
\hline$b$ value & 4.0 & 84.0 & 6.1 & 87.8 & 6.4 & 83.1 & 88.8 \\
\hline
\end{tabular}

Scoring of photopolymerization reactivity, where A: extremely high, B: high, C: low, D: no reactivity. Photo-time: photopolymerization time, NT: not tested, $b$ value of color tone: measured immediately after photopolymerization. Groups from the same column that are identified with the same superscript letter are not significantly different $(p>0.05)$. Full names of CQ-APO, CQ, TMDPO, EDAB, Bis-GMA, and TEGDMA are given in Table 1.

Table 5 Compositions and photopolymerization of experimental, visible light-curing resin composites $^{8)}$

\begin{tabular}{|c|c|c|c|c|c|}
\hline $\begin{array}{l}\text { Ingredients } \\
\text { (parts by weight) }\end{array}$ & CR-1 & CR-2 & CR-3 & CR-4 & CR-5 \\
\hline CQ-APO & 2.5 & 2.5 & 2.5 & 2.5 & 2.5 \\
\hline Tin-Lau & 1.5 & 1.5 & 1.5 & 1.5 & 1.5 \\
\hline TMDPO & - & 0.5 & 1.0 & - & 0.5 \\
\hline UDMA & 60.0 & 60.0 & 60.0 & - & - \\
\hline Bis-GMA & - & - & - & 60.0 & 60.0 \\
\hline TMPT & 40.0 & 40.0 & 40.0 & 40.0 & 40.0 \\
\hline 4-AET & 0.5 & 0.5 & 0.5 & 0.5 & 0.5 \\
\hline Silica filler & 271.4 & 271.4 & 271.4 & 271.4 & 271.4 \\
\hline Fine particle filler & 8.4 & 8.4 & 8.4 & 8.4 & 8.4 \\
\hline Photo-time (sec) & $14.4(1.5)^{\mathrm{a}}$ & $9.6(0.8)^{b}$ & $10.5(0.8)^{b}$ & $10.8(0.5)^{b}$ & $8.8(0.5)^{b}$ \\
\hline Operation time (sec) & $150.0(15)^{\alpha}$ & $150.0(15)^{\alpha}$ & $140.0(10)^{\alpha}$ & $140.0(15)^{\alpha}$ & $130.0(15)^{\alpha}$ \\
\hline Flexural strength (MPa) & $114.3(10.2)^{\mathrm{a}}$ & $122.1(11.3)^{\mathrm{a}}$ & $113.5(12.2)^{\mathrm{a}}$ & $124.5(12.2)^{\mathrm{a}}$ & $133.8(13.5)^{\mathrm{a}}$ \\
\hline Flexural modulus (GPa) & $10.2(1.3)^{\alpha}$ & $11.6(1.5)^{\alpha}$ & $12.3(1.6)^{\alpha}$ & $11.8(1.8)^{\alpha}$ & $13.5(2.1)^{\alpha}$ \\
\hline$b$ value & 16.7 & 18.3 & 18.7 & 17.5 & 18.6 \\
\hline
\end{tabular}

Photo-time: photopolymerization time, $b$ value of color tone: measured immediately after photopolymerization. Groups from the same column that are identified with the same superscript letter are not significantly different ( $p>0.05)$. Full names of Tin-Lau, UDMA, TMPT, CQ-APO, TMDPO and Bis-GMA are given in Table 1. 
photoinitiation mechanism of CQ-APO, it is expedient to perform quantum yield measurement by ESR spectroscopy.

Relationship between color tone and molecular structure On the relationship between color tone and molecular structure for CQ-APO and CQ, Fig. 14 illustrates their stereochemical structures from the views of the $\alpha$ diketone group $[-\mathrm{C}(=\mathrm{O})-\mathrm{C}(=\mathrm{O})-]$ indicated by arrows a and $b^{8)}$. In a stable structure in potential energy, the $\alpha$-diketone group of CQ was given a plane structure (Fig. 14b). However, the $\alpha$-diketone group of CQ-APO had a slightly strained structure (Fig. 14a). It was thought that the slightly strained structure of $\alpha$ diketone group in CQ-APO might be instrumental in yielding a small $b$ value for the yellow shade.

Photopolymerization reactivity, photopolymerization time, operation time, flexural strength and flexural modulus

Physically mixed photoinitiators, namely CQ-APO/TinLau and CQ-APO/Tin-Lau/TMDPO which acted synergistically, exhibited short photopolymerization time and a longer operation time (Table 5). In particular, CR-5 - which contained the novel ternary photoinitiator, CQ-APO/Tin-Lau/TMDPO_ exhibited the highest flexural strength $(133.8 \mathrm{MPa})$ and flexural modulus (13.5 GPa) (Table 5).

Due to the presence of CQ-APO, CQ-APO/Tin-Lau and CQ-APO/Tin-Lau/TMDPO not only underwent $\alpha$ cleavage type of photoinitiation, but also hydrogen abstraction type of photoinitiation with electron donating agents (i.e., tertiary amine or dibutyltin dilaurate), to generate several kinds of free radicals. Consequently, it was achieved an invention termed as "Camphorquinone derivative having acylphosphine oxide group, and containing the same, photopolymerization catalyst and photo/chemical polymerization catalyst, and containing these, hardenable composition" ${ }^{39)}$.

As aliphatic acyldiphenylphosphine oxides are unstable in hydrolytic conditions ${ }^{40)}$, CQ-APO was formulated with Bis-GMA as a base resin in our research study ${ }^{8}$, whereby Bis-GMA is a hydrophobic radical polymerizable monomer. Further, CQ-APO without benzoyl moiety would be a more favorable candidate in improving biocompatibility than TMDPO which releases 2,4,6-trimethylbenzoyl radicals.

\section{EFFECT OF WATER-SOLUBLE PHOTOINITIATOR, QTX, IN SELF-ETCHING ALL-IN-ONE ADHESIVE ON ADHESION}

Invention of a water-soluble photoinitiator QTX for water-based dental adhesives

Masuhara et al..$^{9,10)}$ found that water-reactive TBB initiator enabled hydrophobic MMA to adhere strongly to moist adherends while MMA monomer has $21 \mathrm{vol} \%$ shrinkage ${ }^{41)}$. In light of this finding, several research efforts have focused on designing and developing water-
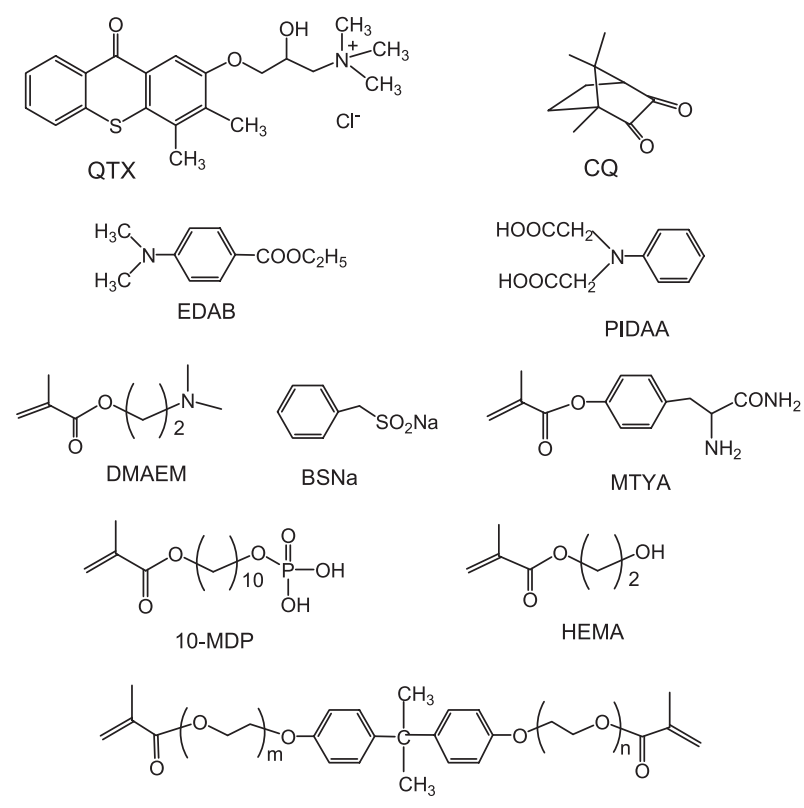

BPE $(m+n=30, M w=1324)$<smiles>[R][CH]C(O)COC(=O)C(=C)C</smiles><smiles>C=CC(=O)OCC(O)COC(=O)C(=C)C</smiles>

Fig. 15 Chemical structures of materials used in the studies $^{12-15)}$ by Hayakawa et al. Full names of QTX, CQ, EDAB, PIDAA, DMAEM, BSNa, MTYA, 10-MDP, HEMA, BPE, GMR, and GAM are given in Table 1.

soluble photoinitiators with an initiation performance like TBB initiator ${ }^{9,10)}$.

In 1986, Davis et al. ${ }^{42)}$ synthesized a series of novel water-soluble thioxanthone photoinitiators. Following this development ${ }^{42)}$, water-based self-etching adhesives containing water-soluble, visible light photoinitiators ${ }^{11-18)}$ have been extensively investigated and reported -in particular, 2-hydroxy-3-(3,4-dimethyl9-oxo-9H-thioxanthen-2-yloxy)-N,N,N-trimethyl-1propanaminium chloride (QTX) ( $\lambda_{\max }=$ circa $500 \mathrm{~nm}$ ). Hayakawa et al. ${ }^{11-16)}$ reported that water-soluble QTX in self-etching adhesives provided efficient adhesion to polished dentin, and their research work culminated in the invention of a water-based photocurable adhesive composition comprising $\mathrm{QXT}^{14)}$.

Effect of water-soluble photoinitiator QTX with adhesive monomers on adhesion

Figure 15 shows the chemical structures of QTX, amines, and monomers employed in the series of QTX studies by Hayakawa et al. ${ }^{12-15)}$. In their early study ${ }^{12)}$, QTX was incorporated in aqueous bonding resin comprising methacryloyl tyrosine amide (MTYA) and glutaraldehyde (GA), and a tensile bond strength of 12 $\mathrm{MPa}$ was obtained after 5 minutes of application on polished dentin.

In their subsequent studies, Hayakawa et al. ${ }^{13-15)}$ 
Table 6 Effect of photoinitiator addition to self-etching, $30 \mathrm{M}$ primer on adhesion of resin composite to dentin and enamel ${ }^{13)}$

\begin{tabular}{ccc}
\hline Photoinitiator (concentration: wt\%) & \multicolumn{2}{c}{ Tensile bond strength (mean \pm SD, MPa) } \\
\cline { 2 - 3 } & Dentin & $13.7(4.9) \omega$ \\
without photoinitiator & $7.8(3.0)^{\mathrm{a}}$ & $13.5(5.3) \omega$ \\
CQ $(0.5)$ & $6.0(3.2)^{\mathrm{a}}$ & $14.6(5.1) \omega$ \\
CQ $(1.0)$ & $7.3(3.8)^{\mathrm{a}}$ & $11.4(4.9) \omega$ \\
CQ (3.0) & $12.8(5.4)^{\mathrm{b}}$ & $12.8(5.3) \omega$ \\
QTX $(0.3)$ & $11.8(8.4)^{\mathrm{b}}$ & $14.2(5.2) \omega$ \\
QTX $(0.5)$ & $7.2(3.1)^{\mathrm{a}}$ & $16.3(6.3) \omega$ \\
QTX (3.0) & $5.6(3.5)^{\mathrm{a}}$ & \\
QTX (5.0)
\end{tabular}

$n=10$ for each treatment. $30 \mathrm{M}$ primer: $30 \mathrm{wt} \%$ 10-MDP/35 wt\% HEMA/35 wt\% water (pH: 1.02), SD: standard deviation. Groups from the same column that are identified with the same superscript letter (a, b, or $\omega)$ are not significantly different ( $p>0.05)$. Full names of QTX, CQ and 10-MDP are given in Table 1.

Table 7 Compositions of self-etching primer adhesives containing QTX ${ }^{15)}$

\begin{tabular}{|c|c|c|c|c|c|c|c|c|}
\hline \multirow{2}{*}{$\begin{array}{l}\text { Abbreviation of } \\
\text { adhesives }\end{array}$} & \multirow[t]{2}{*}{ 10-MDP } & \multirow[t]{2}{*}{ HEMA } & \multicolumn{3}{|c|}{ Water-soluble dimethacrylates } & \multirow[b]{2}{*}{ QTX } & \multicolumn{2}{|c|}{ Amines } \\
\hline & & & $\mathrm{BPE}$ & GMR & GAM & & EDAB & PIDAA \\
\hline $\mathrm{BPE}$ & 5 & 35 & 30 & - & - & 3 & 0.5 & - \\
\hline GMR & 5 & 35 & - & 30 & - & 3 & 0.5 & - \\
\hline GAM & 5 & 35 & - & - & 30 & 3 & 0.5 & - \\
\hline GMR-PIDAA & 5 & 35 & - & 30 & - & - & - & 0.5 \\
\hline
\end{tabular}

Full names of 10-MDP, HEMA, BPE, GMR, GAM, QTX, EDAB, and PIDAA are given in Table 1.

Table 8 Tensile bond strengths of resin composite to bovine dentin and enamel with different self-etching QTXcontaining primer adhesives (BPE, GMR, GAM, and GMR-PIDAA) ${ }^{15}$

\begin{tabular}{ccc}
\hline Self-etching primer adhesive & \multicolumn{2}{c}{ Tensile bond strength (mean \pm SD, MPa) } \\
\cline { 2 - 3 } & Dentin & Enamel \\
\hline BPE & $12.3(5.6)^{, \delta}$ & $14.3(2.4)^{\omega, \delta} \delta$ \\
GMR & $13.2(3.3)^{\mathrm{a}, \delta}$ & $17.2(6.1)^{\omega, \delta}$ \\
GAM & $11.3(3.3)^{\mathrm{a}, \delta}$ & $16.1(5.8)^{\omega, \delta}$ \\
GMR-PIDAA & $19.8(5.2)^{\mathrm{b}, \delta}$ & $20.8(6.4)^{\omega, \delta}$ \\
\hline
\end{tabular}

$n=10$ for each treatment, SD: standard deviation. Groups from the same column that are identified with the same superscript letters $(\mathrm{a}, \mathrm{b}$, or $\omega)$ are not significantly different $(p>0.05)$. Letter $\delta$ indicates mean values that are not significantly different between dentin and enamel for the same self-etching primer adhesive treatment ( $p>0.05)$. Full names of QTX, BPE, GMR and GAM are given in Table 1.

further investigated the effect of the dual presence of QTX and acidic adhesive monomers in self-etching primers on adhesion to smear layer-covered polished dentin. Self-etching primers containing $30 \mathrm{wt} \% 10$ methacryloyloxydecyl dihydrogen phosphate (10-MDP), 35 wt $\%$ HEMA, and 35 wt\% water $(30 \mathrm{M} ; \mathrm{pH}$ value $=1.02$ ) were thus prepared, containing either 0.5-3.0 wt\% CQ or 0.3-5.0 wt\% $\mathrm{QTX}^{13)}$.

Table 6 presents the tensile bond strengths of resin composite to polished bovine dentin and enamel using the self-etching adhesives ${ }^{13)}$. The addition of hydrophobic CQ to $30 \mathrm{M}$ self-etching primer did not increase the bond strength to dentin. However, the addition of water-soluble QTX at $0.3 \mathrm{wt} \%$ and $0.5 \mathrm{wt} \%$ significantly $(p<0.05)$ increased the dentin bond strength to $12.8 \mathrm{MPa}$ and $11.8 \mathrm{MPa}$ respectively. On bond strength to enamel, neither the addition of CQ nor QTX to the self-etching primer (30 M) brought about any significant increase.

Effects of water-soluble dimethacrylates and amines in QTX-containing all-in-one adhesive on adhesion

According to the formulations listed in Table $7^{15}$, experimental all-in-one adhesives were prepared using different combinations of glycerol dimethacrylate (GMR) or glycerol methacrylate acrylate (GAM), ethyl $p$-( $N, N$-dimethylamino)benzoate (EDAB), $N$ phenyliminodiacetic acid (PIDAA), 2,2-bis(4methacryloyloxy polyethoxyphenyl)propane (BPE), 10MDP, HEMA, and QTX.

Table 8 presents the tensile bond strengths of resin composite to tooth substrates using these experimental QTX-containing adhesives. Differences in water-soluble dimethacrylate monomers did not produce any 
significant differences in tensile bond strength to dentin or enamel. The use of PIDAA instead of EDAB showed significantly higher bond strength to dentin and enamel. In particular, the tensile bond strength of GMR-PIDAA to dentin (19.8 $\pm 5.2 \mathrm{MPa})$ was significantly higher than that of GMR to dentin $(13.2 \pm 3.3 \mathrm{MPa})$ $(p<0.05)$. For enamel adhesion, there were no significant differences in tensile bond strength between GMR-PIDAA and GMR ( $p>0.05)$, but the highest bond strength of $20.8 \pm 6.4 \mathrm{MPa}$ to polished enamel was obtained with GMR-PIDAA ${ }^{15)}$. Therefore, the combination of PIDAA and QTX was effective in improving the bond strength of resin composite to dentin and enamel in an all-in-one bonding system, and water-soluble GMR and GMA were useful as a component of the original primer adhesive ${ }^{16)}$.

On the bonding mechanism to dentin, it was suggested that QTX diffused into the moist dentin substrate and initiated an in situ photopolymerization, where the acidic adhesive monomers interacted with the polished dentin substrate to form stronger adhesion as compared to the hydrophobic CQ/tertiary amine system $^{13-15)}$.

Since tertiary amines (i.e., PIDAA and EDAB) accelerated the photopolymerization process ${ }^{15,18)}$, it was probable that QTX performed a hydrogen abstraction initiation mechanism similar to that of $\mathrm{CQ} /$ tertiary amine system. It was thus anticipated that tertiary amines ( $\pi$ acceptor) could react with acidic groups $(\pi$ donor) (i.e., phosphoric acid, phosphonic acid, and carboxyl groups) of adhesive monomers to form

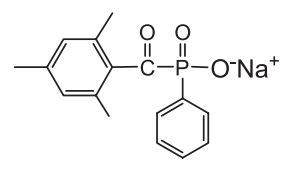

TMPO-Na (=APO-Na)

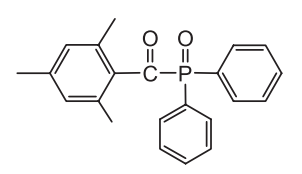

TMDPO (=APO)

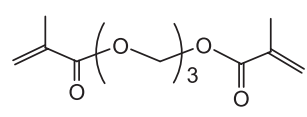

TEGDMA

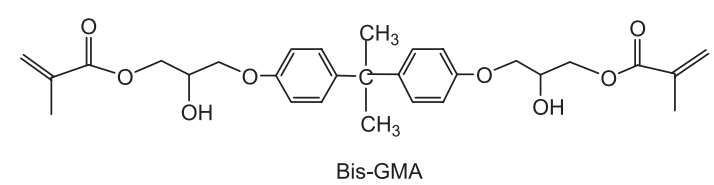

Fig. 16 Structural formulas of the materials employed in the study ${ }^{18)}$ by Ikemura et al. Full names of TMPO-Na (=APO-Na), TMDPO (=APO), 6-MHPA, TEGDMA, 4-MET, and Bis-GMA are given in Table 1. undesirable quaternary ammonium salts ${ }^{4)}$ or chargetransfer complexes (CT complexes) with adverse effects $^{22,23)}$ on initiation reactivity and adhesion.

\section{EFFECT OF A WATER-SOLUBLE PHOTOINITIATOR, APO-NA, WITH CROWN ETHER ON ADHESION}

Design of hydrophobic water-free adhesives comprising water-soluble APO-Na with crown ether

In previous studies ${ }^{11-18)}$ on water-soluble photoinitiators, the focus was almost exclusively on QTX in waterbased self-etching adhesives. In our research study, the focus was shifted to a water-soluble photoinitiator, sodium acylphosphine oxide (APO-Na), in water-free hydrophobic adhesives ${ }^{19)}$.

The behavior of water-soluble photoinitiators with crown ethers in dental adhesives was an unknown. Our recent study ${ }^{19)}$ thus aimed to investigate the effect of APO-Na with crown ether in a hydrophobic adhesive on adhesion to teeth.

APO-Na was synthesized by the reaction between sodium iodide (NaI) and 2,4,6-trimethylbenzoylmethoxy-phenylphosphine oxide (TMMPO), which was synthesized by Michaelis-Arbuzov reaction ${ }^{37,38)}$ between 2,4,6-trimethylbenzoyl chloride (TMC) and dimethoxyphenyl phosphine (DMPP). To dissolve APO$\mathrm{Na}$ as a sodium salt in hydrophobic water-free resins, crown ethers known as clathrate or inclusion compounds were considered. Figure 16 shows the structural formulas of the materials used in the study $^{19)}$.

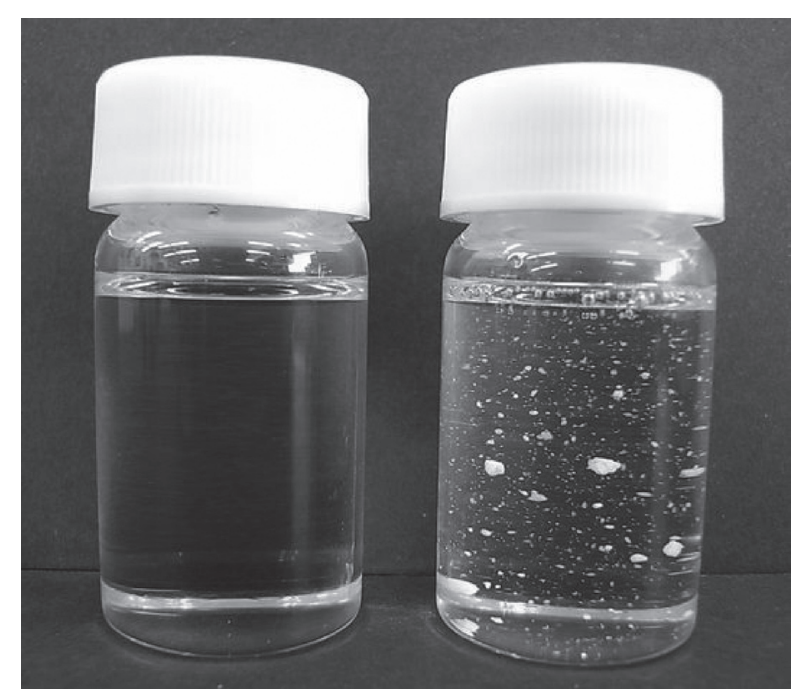

Fig. 17 A photograph of two unfilled resins with (left: Resin A) and without (right: Resin B) 18-crown$6^{19)}$. It is apparent that APO-Na powder was dissolved in hydrophobic Bis-GMA/TEGDMA resin in the presence of crown ether to form a clear liquid (Resin A). On the contrary, Resin B without crown ether appears turbid because the white powder of APO-Na was not dissolved in hydrophobic resin. 
Table 9 Effect of the concentration of crown ether in experimental, one-bottle, single-step bonding resins on shear bond strength (SBS) to unetched ground enamel and dentin (Method A) ${ }^{19)}$

\begin{tabular}{lccccrr}
\hline $\begin{array}{l}\text { 6-MHPA } \\
\text { /4-MET } \\
\text { (wt\%) }\end{array}$ & $\begin{array}{c}\text { Bis-GMA/ } \\
\text { TEGDMA (60/40) } \\
(\mathrm{wt} \%)\end{array}$ & $\begin{array}{c}\text { APO-Na } \\
(\mathrm{wt} \%)\end{array}$ & $\begin{array}{c}\text { CE-6 } \\
\text { (wt\%) }\end{array}$ & $\begin{array}{c}\text { Acetone } \\
\text { (wt\%) }\end{array}$ & $\begin{array}{c}\text { SBS } \\
\text { to Enamel } \\
{[\mathrm{MPa}(\mathrm{SD})]}\end{array}$ & $\begin{array}{c}\text { SBS } \\
\text { to Dentin } \\
{[\mathrm{MPa}(\mathrm{SD})]}\end{array}$ \\
\hline $15.0 / 20.0$ & 35.0 & 0.50 & - & 29.50 & $2.3(1.2)^{\mathrm{a}}$ & $2.5(0.8)^{\mathrm{a}}$ \\
$15.0 / 20.0$ & 35.0 & 0.50 & 0.25 & 29.25 & $11.3(3.4)^{\mathrm{b}}$ & $12.8(4.2)^{\mathrm{b}}$ \\
$15.0 / 20.0$ & 35.0 & 0.50 & 0.50 & 29.00 & $13.6(3.3)^{\mathrm{b}}$ & $14.1(3.6)^{\mathrm{b}}$ \\
$15.0 / 20.0$ & 35.0 & 0.50 & 1.00 & 28.50 & $8.2(3.8)^{\mathrm{b}}$ & $10.5(4.7)^{\mathrm{b}}$ \\
$15.0 / 20.0$ & 35.0 & APO: 0.50 & - & 29.50 & $7.4(2.4)^{\mathrm{b}}$ & $5.3(2.6)^{\mathrm{c}}$ \\
\hline
\end{tabular}

$n=10$, SD: standard deviation. Method A: One-bottle single-step adhesive, where bonding resin was applied to ground teeth. Groups from the same column that are identified with the same superscript letter are not significantly different $(p>0.05)$. Full names of 6-MHPA, 4-MET, Bis-GMA, TEGDMA, APO-Na (=TMPO-Na), and CE-6 are given in Table 1.

Table 10 Effect of the concentration of acidic adhesive monomers (6-MHPA and 4-MET) in experimental, two-bottle, single-step bonding resins on shear bond strength (SBS) to unetched ground enamel and dentin (Method B) ${ }^{19)}$

\begin{tabular}{cccccccc}
\hline $\begin{array}{l}6 \text {-MHPA } \\
(\mathrm{wt} \%)\end{array}$ & $\begin{array}{c}4 \text {-MET } \\
(\mathrm{wt} \%)\end{array}$ & $\begin{array}{c}\text { Bis-GMA/ } \\
\text { TEGDMA (60/40) } \\
(\mathrm{wt} \%)\end{array}$ & $\begin{array}{c}\text { APO-Na } \\
(\mathrm{wt} \%)\end{array}$ & $\begin{array}{c}\text { CE-6 } \\
\text { (wt\%) }\end{array}$ & $\begin{array}{c}\text { Acetone } \\
\text { (wt\%) }\end{array}$ & $\begin{array}{c}\text { SBS } \\
\text { to Enamel } \\
{[\mathrm{MPa}(\mathrm{SD})]}\end{array}$ & $\begin{array}{c}\text { SBS } \\
\text { to Dentin } \\
{[\mathrm{MPa}(\mathrm{SD})]}\end{array}$ \\
\hline 0 & 0 & 70.0 & 0.5 & 0.5 & 29.0 & $2.2(0.8)^{\mathrm{a}}$ & $1.1(1.5)^{\mathrm{b}}$ \\
12.0 & 16.0 & 42.0 & 0.5 & 0.5 & 29.0 & $17.1(3.8)^{\mathrm{b}}$ & $15.8(4.3)^{\mathrm{b}}$ \\
15.0 & 20.0 & 35.0 & 0.5 & 0.5 & 29.0 & $17.9(3.5)^{\mathrm{b}}$ & $14.1(3.8)^{\mathrm{b}}$ \\
18.0 & 24.0 & 28.0 & 0.5 & 0.5 & 29.0 & $16.5(3.9)^{\mathrm{b}}$ & $15.5(4.6)^{\mathrm{b}}$ \\
21.0 & 28.0 & 21.0 & 0.5 & 0.5 & 29.0 & $16.3(4.2)^{\mathrm{b}}$ & $20.2(4.7)^{\mathrm{b}}$ \\
\hline
\end{tabular}

$n=10$, SD: standard deviation. Method B: Two-bottle single-step adhesive, where bonding resin was mixed with PTS solution ( $p$-TSNa/acetone/water=3.0/27/70 wt\%) and applied to ground teeth. Groups from the same column that are identified with the same superscript letter are not significantly different $(p>0.05)$. Full names of 6-MHPA, 4-MET, APONa, CE-6, Bis-GMA, and TEGDMA are given in Table 1.

Ionophor effect of crown ethers and optimal concentration of APO-Na and crown ether

An outstanding characteristic of crown ethers is their ability to embed inorganic cations (i.e., APO-Na) or amino acids (in $\mathrm{NH}_{3}^{+}$) in their cavity, hence they are known for their ability to strongly solvate cations. This is the so-called ionophore effect ${ }^{43)}$ shown in Fig. 17, which enabled water-soluble APO-Na to dissolve in hydrophobic adhesive solutions in the presence of crown ether.

Experimental water-free adhesives comprising APO-Na, CE, Bis-GMA, 6-methacryloyloxyhexyl phosphonoacetate (6-MHPA), and methacryloyloxyethyltrimellitic acid (4-MET) were prepared. Their compositions are shown in Tables 9 and 10. Shear bond strengths of resin composites with these adhesives to unetched ground teeth were measured at a crosshead speed of $1.0 \mathrm{~mm} / \mathrm{min}$, and the data were analyzed by ANOVA.

Table 9 presents the effects of the concentration of 18-crown-6-ether (CE-6) with APO-Na in one-bottle bonding resins on adhesion to unetched-ground enamel and dentin. One-bottle bonding resin was directly applied to ground enamel or dentin as a one-bottle, single-step, bonding resin (Method A). Based on the shear bond strength results shown in Table 9, the optimal concentration was determined to be $0.5 \mathrm{wt} \%$ for both APO-Na and CE-6, at which the highest bond strength values were obtained for ground enamel $(13.6 \pm 3.3 \mathrm{MPa})$ and ground dentin $(14.1 \pm 3.6 \mathrm{MPa})$.

Effect of adhesive monomers in APO-Na-containing adhesives on adhesion and bonding mechanism

Table 10 presents the effects of the concentration of acidic adhesive monomers (6-MHPA and 4-MET) with APO-Na and CE- 6 in the bonding resins on adhesion to unetched ground enamel and dentin. PTS-solution [a mixture of $p$-TSNa/acetone/water=3.0/27/70 (wt\%)] was employed for Method B (two-bottle, single-step).

It was apparent that the shear bond strengths of 6-MHPA- and 4-MET-containing bonding resins to both ground enamel and dentin were significantly higher than that without these adhesive monomers $(p<0.05)$. For bonding to enamel, highest shear bond (17.9 \pm 3.5 $\mathrm{MPa})$ was achieved with the adhesive containing 15.0 wt\% 6-MHPA/20.0 wt\% 4-MET; for bonding to dentin, highest shear bond strength $(20.2 \pm 4.7 \mathrm{MPa})$ was achieved with the adhesive containing $21.0 \mathrm{wt} \%$ 6MHPA/28.0 wt\% 4-MET. These results confirmed that water aptly controlled and regulated the bonding function of adhesive monomers to the tooth substrates. These findings eventually culminated in the invention termed as "A curable composition comprising clathrate compounds" 4 . 
6-MHPA and 4-MET are known as an amphiphilic molecules that have both hydrophilic and hydrophobic parts. On the underlying bonding mechanism to the tooth substrates, it was hypothesized based on the theory of Langmuir-Blodgett (LB) films ${ }^{45}$ ) that the ionized hydrophilic parts [i.e., $-\mathrm{P}(=\mathrm{O})\left(\mathrm{O}^{-}\right)_{2}, \quad$-COOinteracted with hydroxyapatite in the tooth substrates, wherein ionized APO-Na $\left(-\mathrm{O}^{-} \mathrm{Na}^{+}\right)$then initiated in situ photopolymerization at the resin-tooth interface to improve adhesion.

\section{Stability of APO-Na and biological safety of crown ethers}

On photoinitiator stability, APO and BAPO derivatives are generally prone to undergoing solvolytic cleavage of the $\mathrm{C}-\mathrm{P}$ bond $[>\mathrm{P}(\mathrm{C}=\mathrm{O})-(\mathrm{C}=\mathrm{O})-]$ in the presence of nucleophilic compounds, such as water. However, it has been shown that the solvolytic stability of acylphosphine oxides could be greatly enhanced by the substituent effect of stereochemistry, where the presence of methyl group in the ortho positions enabled the carbonyl group to be shielded from nucleophilic $\operatorname{attack}^{40)}$.

For this reason, commercially utilized APO and BAPO have trimethylbenzoyl moiety to improve their solvolytic stability ${ }^{5}$ and render them more stable in hydrophobic resins such as Bis-GMA resin. Similarly, due to the presence of trimethylbenzoyl moiety in the structure, TMPO-Na (APO-Na) was stable in Bis-GMA/ TEGDMA resins.

Although APO-Na with crown ether had favorably contributed to efficient adhesion with the tooth substrates, the toxicity of crown ethers must also be duly highlighted at this juncture. On the toxicity of crown ethers, the acute $\mathrm{LD}_{50}$ (lethal dose, 50 percent kill) values in mice of 12-crown-4, 15-crown-5, and 18crown-6 are $3,150 \mathrm{mg} / \mathrm{kg}, 1,020 \mathrm{mg} / \mathrm{kg}$, and $7,100 \mathrm{mg} / \mathrm{kg}$ respectively ${ }^{46)}$.

\section{DEVELOPMENT OF A NEW HEMA-FREE, SELF- ETCHING ADHESIVE WITHOUT USE OF WATER- SOLUBLE PHOTOINITIATORS}

Rationale for developing HEMA-free bonding resins without the use of water-soluble photoinitiators

In our recent study ${ }^{19}$ ) on the effect of APO-Na on adhesion, the experimental bonding resins - unlike conventional self-etching adhesives- contained neither water nor HEMA. Although APO-Na with crown ether markedly improved the bond strength of hydrophobic adhesives to ground enamel and dentin, there remain some major biosafety issues of crown ethers to contend with.

Nonetheless, based on the encouraging results of HEMA-free, APO-Na-containing adhesives toward adhesion $^{19)}$, our next research target was to develop a HEMA-free, self-etching adhesive without the use of water-soluble photoinitiators.
Inherent disadvantages of HEMA for single-bottle adhesives

A number of compromises have to be made when selfetching adhesives are simplified into single-bottle formulations ${ }^{47)}$ that usually contain acidic adhesive monomers, together with both water and water-soluble monomers, such as HEMA.

HEMA has several inherent disadvantages. It retains water within the adhesive layer and causes the mechanical strength to be adversely affected ${ }^{48)} ; 70 \%$ of HEMA could be hydrolyzed in $37^{\circ} \mathrm{C}$ acidic solutions $(\mathrm{pH}$ 1.0) within a week ${ }^{49}$; it causes delayed allergic reactions in sensitized persons ${ }^{50)}$. Since HEMA provides low photopolymerization reactivity ${ }^{27)}$, it also meant that the bonding layer containing HEMA would produce low physical properties with high water absorption characteristic, thereby resulting in poor bonding durability to teeth. Conversely, it was anticipated that a HEMA-free adhesive would produce good physical properties with good bonding durability.

Development of HEMA-free single-bottle bonding resin In our recent study ${ }^{20)}$, the effect of experimental HEMA-free, single-step, self-etching adhesives containing conventional $\mathrm{CQ} /$ tertiary amine initiator on adhesion was further investigated. It was found that the experimental HEMA-free self-etching adhesives adhered strongly to ground enamel and dentin, and an invention entitled "A $\mathrm{pH}$-controlled adhesive composition" ${ }^{51)}$ was achieved.

Recently, a new one-bottle single-step, HEMA-free, self-etching bonding resin BeautiBond (Shofu Inc., Kyoto, Japan) was developed and introduced to the dental market (2008). As a seventh-generation adhesive, BeautiBond exhibits the advantages of being fast and easy to use, with better adhesion to both enamel and dentin in a single step ${ }^{52)}$.

\section{PHOTOINITIATING MONOMERS AND POLYMERIC PHOTOINITIATORS FOR BIOMEDICAL APPLICATION}

\section{Photoinitiators for biomedical applications}

Currently, photoinitiating monomers and polymeric photoinitiators are widely used for biomedical applications such as bone cements and tooth filling materials. In vivo polymerization of injectable prepolymer liquids provides the possibility of forming polymers in complex shapes ${ }^{40)}$. This means that implantation can be performed through small holes and is therefore less invasive than conventional surgical techniques ${ }^{40)}$.

During photopolymerization, insufficient reactivity of photoinitiators will cause a substantial amount of unreacted and less reacted photoinitiator fragments to remain and subsequently migrate out of the photopolymerized material ${ }^{40)}$. To overcome this problem, it is well investigated so-called polymeric photoinitiators which bear photosensitive moieties in the side chains of their polymer chemical structures, 
thereby bestowing their macromolecular structures with important reactivity-related advantages.

Synthesis of visible light photoinitiating monomer containing anthraquinone

Recently, several photoinitiating monomers defined as compounds bearing photoinitiating moiety and radical polymerizable group intramolecularly were developed. They are radical polymerizable photoinitiators, and they act as self-crosslinking species as well as photoinitiators.

Notably, Takeuchi and Mizuta ${ }^{53)}$ invented a photocurable resin composition containing an anthraquinone derivative, which comprised a polymerizable moiety intramolecularly, as a photoinitiating monomer. One of the derivatives (HTAQ-MEI) was synthesized by the reaction between 2-(2-hydroxyethylthio)-9,10-anthraquinone (HTAQ) and 2-methacryloyloxyethyl isocyanate (MEI) (Fig. 18). It was disclosed that HTAQ-MEI exhibited excellent reactivity under both ultraviolet and visible light irradiation and that occurrences of outgassing from hardened materials during photopolymerization were minimal.

Synthesis of visible light-photoinitiated vinyl-functional acylphosphine oxides

Although 2,4,6-trimethylbenzoyl group contributed to the photoinitiator stability of TMDPO initiator, the benzoyl radicals generated as a result of the $\alpha$-cleavage of TMDPO posed a biological hazard ${ }^{40)}$ in biomedical and dental applications.

To develop photoinitiating monomers for biomedical applications, de Groot et al. ${ }^{40)}$ synthesized three kinds of vinyl-functional acylphosphine oxides, as depicted in Fig. 19: 4-vinylbenzoyl diphenylphosphine oxide (VBPO), 2,6-dimethyl-4-vinylbenzoyl-diphenylphosphine oxide (DMVBPO), and 2,4,6-trimethylbenzoyl(phenyl-4vinylphenyl)phosphine oxide (TMBVPO). They were copolymerized with $N$-vinylpyrrolidone (NVP) or dimethylacrylamide (DAM) to form poly(VBPO-co-NVP) [PPI-1a], poly(VBPO-co-DMA) [PPI-1b], poly(DMVBPOco-DAM) [PPI-2] and poly(TMBVPO-co-DAM) [PPI- 3] respectively.

In the case of the above-described photoinitiating monomers, benzoyl radicals bearing a polymerizable group (VBPO or DMVBPO) could copolymerize with coexisting radical polymerizable resin monomers, thereby preventing the migration of benzoyl radicals from the polymers. An additional advantage of these photoinitiating monomers is that they could be activated by visible light.

Visible light reactivity and stability of polymeric photoinitiators bearing APO moiety in HEMA solution

To investigate the photopolymerization of these synthesized polymeric photoinitiators (i.e., PPI-1a, PPI1b, PPI-2, and PPI-3) under blue light (visible light) irradiation, photocalorimetric studies were performed using a DSC (photo-DSC) in combination with a blue

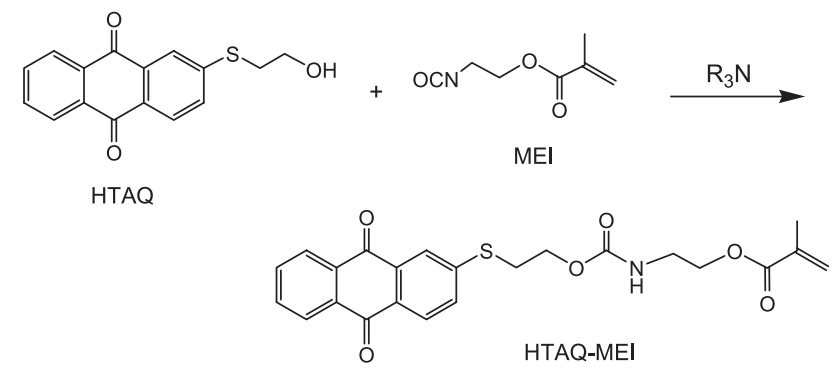

Fig. 18 Synthesis of anthraquinone derivative, which comprised a polymerizable moiety (HTAQ-MEI), by a reaction between 2-(2-hydroxyethylthio)-9,10anthraquinone (HTAQ) and 2methacryloyloxyethyl isocyanate (MEI) ${ }^{53)}$.

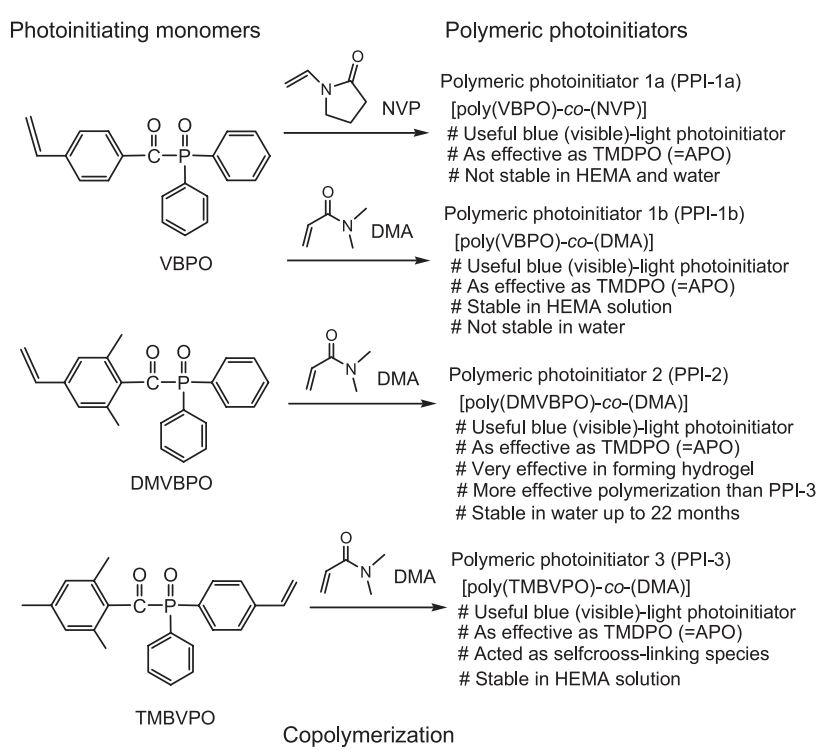

Fig. 19 Structural formulas of vinyl-functional phosphine oxide photoinitiating monomers ${ }^{40)}$. Full names of VBPO, DMVBPO, TMBVPO, and their copolymers with NVP or DAM are given in Table 1.

light dental gun (Heliolux DLX, Vivadent, Liechtenstein). HEMA, photoinitiator, and in some cases, water were mixed in subdued visible light.

Figure 19 also presents the characteristics of these synthesized polymeric photoinitiators. Results showed that all the synthesized polymeric photoinitiators were useful in visible light bleaching photoinitiators. On photoinitiator stability, PPI-1a was not stable in HEMA solution, whereas VBPO-dimethylacrylamide copolymer (PPI-1b) was stable in HEMA but not stable in aqueous solution. PPI-2 was both soluble and stable in water up to 22 months.

On polymerization efficiency, PPI-1a was as effective as trimethylbenzoyl diphenylphosphine oxide (TMDPO). PPI-2 exhibited greater polymerization efficiency in HEMA/water mixtures than PPI-3. PPI-2 and PPI-3 acted as self-crosslinking species, resulting 


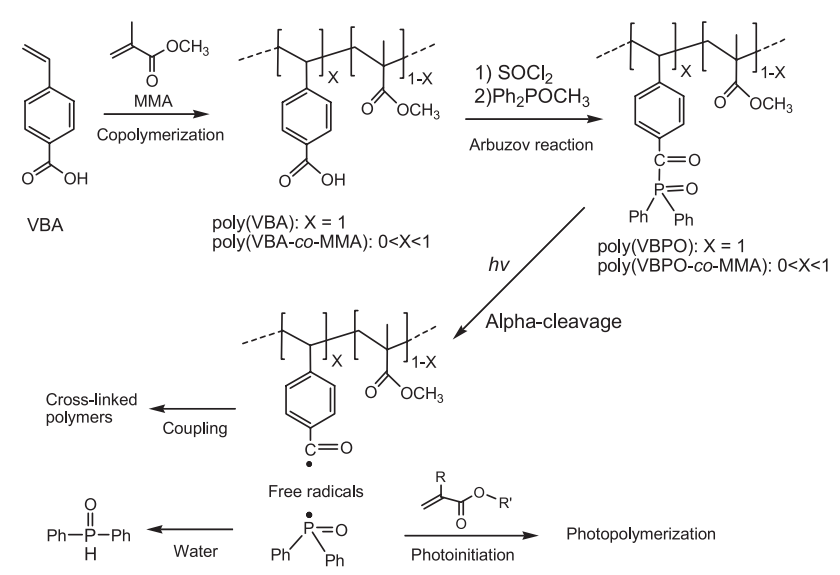

Fig. 20 Structural formula of a polymeric photoinitiator bearing side-chain benzoyl diphenylphosphine oxide moieties and its $\alpha$-cleavage photoinitiation ${ }^{54)}$. Full names of poly(VBPO) and poly(VBPO-coMMA) are given in Table 1.
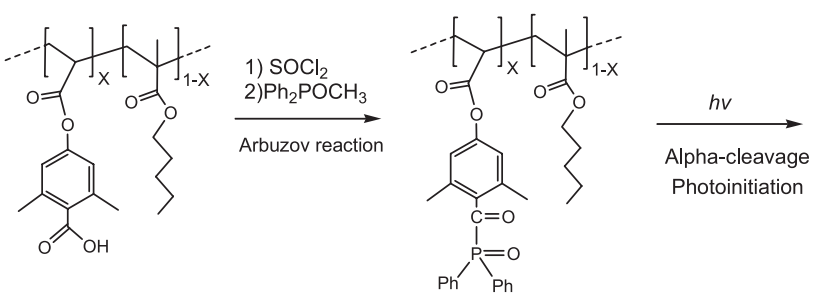

poly(ADBA): $\mathrm{X}=1$ poly(ADBA-CO-BA): $0<X<1$

poly(ADBPO): $X=1$ poly(ADBPO-CO-MMA): $0<X<1$

Fig. 21 Structural formula of a polymeric photoinitiator with pendant 2,6-dimethylbenzoyl-diphenylphosphine oxide moieties ${ }^{55}$. Full names of poly(ADBA) and poly(ADBA-co-BA) are given in Table 1.

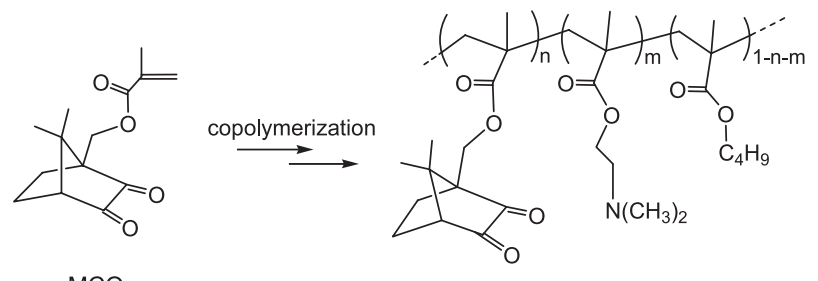

$\mathrm{MCQ}$

$$
\text { poly(MCQ-co-BM-co-DMAEM) }
$$

Fig. 22 Structural formula of radical polymeric photoinitiator bearing camphorquinone moieties ${ }^{1)}$. Full names of MCQ and poly(MCQ-co-BM-coDMAEM) are given in Table 1.

in formation of hydrogels, and that PPI-3 was more effective than PPI-2 in this aspect. PPI-2 was very effective in forming hydrogels based on poly(ethylene glycol) diacrylate.

Synthesis and kinetics of polymeric photoinitiators bearing side-chain APO pendant

Angiolini et al. ${ }^{54)}$ synthesized polymeric photoinitiators bearing side-chain benzoyl diphenylphosphine oxide moieties [poly(VBPO) and poly(VBPO-co-MMA)] prepared by a reaction between poly(4-vinylbenzoic acid) [poly(VBA)] or [poly(VBA-co-MMA)] and thionyl chloride, followed by a reaction with methoxydiphenylphosphine (Fig. 20).

As for polymeric photoinitiators with pendant 2,6dimethylbenzoyl diphenylphosphine oxide moieties [poly(ADBPO) or poly(ADBPO-co-BA)], they were synthesized by reacting homopolymer of 4-acryloyloxy2,6-dimethyl benzoic acid [poly(ADBA)] or $\mathrm{ADBA} / n$ butyl acrylate (BA) copolymers [poly(ADBPO-co-BA)] with thionyl chloride, followed by a reaction with methoxydiphenylphosphine ${ }^{55)}$ (Fig. 21).

On photoinitiation activity, poly(VBPO-co-MMA)s exhibited markedly improved activity than analogous aliphatic polymeric systems bearing the acylphosphine oxide moiety in the side chain ${ }^{54)}$. On stability to daylight, poly(ADBPO-co-BA) exhibited appreciably higher stability than TMDPO, which is reported to be the most stable commercial member of this class of photoinitiators $^{55)}$.

Synthesis and kinetics of polymeric photoinitiators bearing side-chain camphorquinone moiety

In a study by Angiolini et al. ${ }^{1)}$, novel polymethacrylic photoinitiators bearing the photosensitive camphorquinone moiety in the side chain, D,L-10methacryloyloxy-camphorquinone (MCQ) (Fig. 22), was synthesized by the reaction between D,L-10hydroxycamphorquinone and methacryloyl chloride. Monomer MCQ was copolymerized with 2dimethylaminoethyl methacrylate (DMAEM) in different ratios to give the corresponding polymeric derivatives, poly(MCQ) and poly(MCQ-co-DMAEM). To improve solubility, copolymer derivatives poly(MCQ-co$\mathrm{BM}$ ) and poly(MCQ-co-BM-co-DMAEM) were further synthesized using the butyl methacrylate (BM) comonomer.

An equimolar 1,6-hexanediol diacrylate (HDDA)/ butyl acrylate (BA) mixture (50/50, mol\%) containing 1 mol\% of camphorquinone moiety with an equimolar amount of amine was polymerized under a nitrogenrich atmosphere by irradiation from a high-pressure mercury lamp (>400 nm). Table 11 presents the UVVIS properties and kinetic data of the synthesized polymeric photoinitiators bearing the camphorquinone moiety, as a function of the photoinduced crosslinking polymerization.

In terms of UV-VIS spectral data, the maximum absorption wavelengths $\left(\lambda_{\max }\right)$ of CQ, synthesized poly(MCQ-co-DMAEM) and poly(MCQ-co-BM-coDMAEM) were 483,469 , and $469 \mathrm{~nm}$ respectively. The molar extinction coefficients $\left(\varepsilon_{\max }, 1 \mathrm{~mol}^{-1} \mathrm{~cm}^{-1}\right)$ of these photoinitiators were $39.0,35.8$, and 35.6 respectively. Among the investigated photoinitiators, poly(MCQ-co$\mathrm{BM} / \mathrm{DMAEM}$ exhibited the best photoinitiation activity although it had a slightly higher induction time (Table 11).

Nonetheless, for copolymeric photoinitiators 
Table 11 UV-VIS properties and kinetic data of photoinitiation activity by polymeric and low-molecular-weight photoinitiator systems based on camphorquinone in the photopolymerization of an equimolar HDDA/BA mixture upon irradiation above $400 \mathrm{~nm}^{1)}$

\begin{tabular}{llcccc}
\hline Photoinitiators & \multicolumn{2}{c}{ UV-VIS properties } & $T_{\text {ind }}$ (sec) & $t_{1 / 2}$ (sec) & Conversion (\%) \\
& $\lambda_{\max }$ & $\mathcal{E}_{\max }$ & & & \\
\hline CQ & 483 & 39.0 & 63 & 515 & 60.1 \\
Poly(MCQ-co-BM)/DMAEM & 469 & 35.8 & 15 & 64 & 82 \\
Poly(MCQ-co-BM)/poly(DMAEM) & 469 & 35.8 & 25 & 152 & 85.2 \\
Poly(MCQ -co-BM- co-DMAEM) & 469 & 35.6 & 25.4 & 87.0 \\
\hline
\end{tabular}

Photoinitiator concentration in the acrylic formulation: $1.0 \mathrm{~mol} \%$ of camphorquinone moiety with an equimolar amount of amine when used in combination. The values of $\lambda_{\max }$ and $\varepsilon_{\max }$ were not indicative of DMAEM and poly(DMAEP). $T_{\text {ind: }}$ Induction time of the curing process at $1 \%$ conversion of double bond. $t_{1 / 2}$ :Time required for reaching $50 \%$ conversion of the HDDA/BA mixture. Conversion: Double-bond conversion after 600 seconds of irradiation time. Full names of HDDA, BA, CQ, DMAEP, Poly(DMAEM), Poly(MCQ-co-BM), Poly(MCQ -co-BM- co-DMAEM), $\lambda_{\max }$ and $\varepsilon_{\max }$ are given in Table 1.

containing the photoreactive moieties of camphorquinone and tertiary amine in the side chain, the general trend is a decrease in efficiency in terms of induction time and polymerization rate, probably because of steric hindrance to exciplex formation ${ }^{1)}$. The close proximity of the photosensitive moieties to the macromolecular backbone most probably reduced their mobility, thus favoring recombination reactions between the active radical species. Moreover, since an inordinate number of steps are needed for their syntheses, it seemed that these copolymeric photoinitiators would remain limited to industrial application.

In conclusion, for the copolymeric photoinitiator containing both photoreactive camphorquinone and synergistic tertiary amine moieties in the side chain, the efficiency in terms of induction time and polymerization rate decreased, probably because of steric hindrance to exciplex formation ${ }^{1)}$. The close vicinity of the photosensitive moieties to the macromolecular backbone most probably reduced their mobility, thus favoring recombination reactions between the active radical species. Moreover, since an inordinate number of steps are needed for their syntheses, it seemed that these copolymeric photoinitiators would remain limited to industrial application.

Upon completion of this review paper, a myriad of photoinitiators would have been developed. However, the scope and limitation of this paper was to review recent studies on radical photopolymerization initiators, which were used in the design of light-curing dental adhesives and resin composites, by collating information of related studies on these photoinitiators.

\section{CONCLUSION}

Within the limitations of this review paper, the key conclusions drawn are as follows:

1. No significant differences in degree of conversion (DC) $(p>0.05)$ were observed between BAPO and CQ/amine system.
2. Newly synthesized 7,7-dimethyl-2,3-dioxobicyclo [2.2.1]heptane-1-carbonyldiphenyl phosphine oxide (DOHC-DPPO=CQ-APO) possessed two maximum absorption wavelengths $\left(\lambda_{\max }\right)$ at 350 $500 \mathrm{~nm}[372 \mathrm{~nm}$ (from APO group) and $475 \mathrm{~nm}$ (from CQ moiety)].

3. CQ-APO-containing resins exhibited good photopolymerization reactivity, excellent color tone, relaxed operation time, and high mechanical strength.

4. A water-soluble photoinitiator APO-Na with crown ether improved the adhesion of single-step hydrophobic bonding resin to ground dentin, and an experimental HEMA-free self-etching adhesive exhibited strong adhesion to both ground enamel and dentin.

5. For copolymeric photoinitiators containing the photoreactive moieties of camphorquinone and tertiary amine in the side chain, there was a discernible decrease in efficiency in terms of induction time and polymerization rate, probably because of steric hindrance to exciplex formation.

\section{ACKNOWLEDGMENTS}

This review paper was included as a part of Dr. Ikemura's lecture (in English) entitled "Development of dental adhesives and their bonding mechanism to dentin" for Ph.D students in Graduate Course of Cariology and Operative Dentistry, Department of Restorative Sciences, Graduate School, Tokyo Medical and Dental University (Dr. Junji Tagami, Professor and Chair) for 15 years (1995-2009). The authors thank Professor Junji Tagami who kindly gave us the opportunity to submit this article to a refereed scientific journal for the preparation of a course textbook for $\mathrm{Ph}$. D students.

The authors also thank Mr. Noriyuki Negoro, President of Shofu Inc., Kyoto, Japan, and all research co-workers at the Department of Research and Development of Shofu Inc, Kyoto, Japan, for their helpful efforts and discussions in the preparation of 
this review paper.

\section{REFERENCES}

1) Angiolini L, Caretti D, Salatelli E. Synthesis and photoinitiation activity of radical polymeric photoinitiators bearing side-chain camphorquinone moieties. Macromol Chem Phys 2000; 201: 2646-2653.

2) Wataha JC, Lockwood PE, Lewis JB, Rueggeberg FA, Messer RLW. Biological effects of blue light from dental curing units. Dent Mater 2004; 20: 150-157.

3) Dart EC, Nemcek J. Photopolymerisable composition. Great Britain Patent Specification No. 1408265 (1971); Japanese Patent No. Toku-Kou-Sho 54-10986 (1979).

4) Tay FR, King NM, Suh BI, Pashley DH. Effect of delayed activation of light-curing resin composites on bonding of allin-one adhesives. J Adhes Dent 2001; 3: 207-225.

5) Jockusch S, Koptyug IV, McGarry PF, Sluggett GW, Turro NJ, Watkins DW. A steady-state and picosecond pumpprobe investigation of the photophysics of an acyl and a bis(acyl)phosphine oxide. J Am Chem Soc 1996; 119: 1149511501.

6) Sumiyoshi T, Schnabel W, Henne A, Lechtken P. On the photolysis of acylphosphine oxides: 1. Laser flash photolysis studies with 2,4,6-trimethylbenzoyldiphenylphosphine oxide. Polymer 1985; 26: 141-146.

7) Arikawa H, Takahashi H, Kanie T, Ban S. Effect of various visible light photoinitiators on the polymerization and color of light-activated resins. Dent Mater J 2009; 28: 454-460.

8) Ikemura K, Ichizawa K, Jogetsu Y, Endo T. Synthesis of a novel camphorquinone derivative having acylphosphine oxide group, characterization by UV-VIS spectroscopy and evaluation of photopolymerization performance. Dent Mater J 2009; 29: 122-131.

9) Masuhara E, Kojima K, Hirasawa T, Tarumi N, Kimura T. Studies on dental self-curing resins. 3. Effects of alkylboran on the adhesive force to ivory and tooth surface. Rep Inst Dent Mater Tokyo Med Dent Univ 1963; 2: 457-456.

10) Masuhara E. Über die Chemie eines neuen haftfähigen Kunststoff-Füllungsmaterials. DZZ 1969; 24: 620-628.

11) Hayakawa T, Horie K. Effect of water-soluble photoinitiator on the adhesion between composite and tooth substrate. Dent Mater J 1992; 8: 351-353.

12) Hayakawa T, Nemoto K, Horie K. Adhesion of composite to polished dentin retaining its smear layer. Dent Mater 1995; 11: $218-222$

13) Hayakawa T, Kikutake K, Nemoto K. Effectiveness of the addition of water-soluble photoinitiator into the self-etching primers on the adhesion of a resin composite to polished dentin and enamel. Dent Mater J 1999; 18: 324-333.

14) Hayakawa T, Kidokoro N. Water-based photo-curable adhesive composition. Japanese Patent No. Toku-Kou$3158422(2001)$

15) Hayakawa T, Kikutake-Sugiyama K, Fukushima T, Nemoto K. Development of self-etching primer adhesive in all-inone bonding system. Dent Mater J 2005; 24: 251-256.

16) Hayakawa T, Kikutake-Sugiyama K, Nemoto K. Efficacy of water-soluble photoinitiator on the adhesion of composite resin to bovine teeth in all-in-one bonding system. Dent Mater J 2005; 24: 213-218.

17) Kikutake K, Hayakawa T, Nemoto K. Study on the selfetching primer containing water-soluble photoinitiator. Adhes Dent 1998; 16: 152-160.

18) Kikutake K. Development of a one step adhesion system using photo curing self-etching primer. J J Dent Mater 1999; 18: 151-169.

19) Ikemura K, Ichizawa K, Fuchigami K, Ito S, Endo T. Design of a new dental adhesive - Effect of a water-soluble sodium acylphosphine oxide with crown ether on adhesion to dental hard tissues. Dent Mater J 2009; 28: 267-276.

20) Ikemura K, Ichizawa K, Endo T. Design of a new selfetching HEMA-free adhesive. Dent Mater J 2009; 28: 558564 .

21) Ikemura K, Endo T. A review of our development of dental adhesives - Effects of radical polymerization initiators and adhesive monomers on adhesion. Dent Mater J 2010; 29: 109-121.

22) Bowen RL, Cobb EN, Rapson JE. Adhesive bonding of various materials to hard tooth tissues: Improvement in bond strength to dentin. J Dent Res 1982; 61: 1070-1076.

23) Ikemura K, Endo T. Effect on adhesion of new polymerization initiator systems comprising 5monosubstituted barbituric acids, aromatic sulfinate amides and tert-butylperoxymaleic acid in dental adhesive. J Appl Polym Sci 1999; 72: 1655-1668.

24) Lechtken PI, Jacobi M, Trimborn W. Acylphosphine oxide compounds: their preparation and use. United States Patent No. 4298738; 1981.

25) Kolczak U, Rist G, Dietliker K, Wirz J. Reaction mechanism of monoacyl- and bisacylphosphine oxide photoinitiators studied by ${ }^{31} \mathrm{P}-,{ }^{13} \mathrm{C}-$, and ${ }^{1} \mathrm{H}-\mathrm{CIDNP}$ and ESR. J Am Chem Soc 1996; 118: 6477-6489.

26) Rutsch W, Dietliker K, Leppard D, Kohler M, Misev L, Kolczak U. Recent developments in photoinitiators. Progress in Organic Coatings 1996; 27: 227-239.

27) Ikemura K, Ichizawa K, Yoshida M, Ito S, Endo T. UV-VIS spectra and photoinitiation behaviors of acylphosphine oxide and bisacylphosphine oxide derivatives in unfilled, lightcuring dental resins. Dent Mater J 2008; 27: 765-774.

28) Atai M, Watts DC. A new kinetic model for the photopolymerization shrinkage-strain of dental composites and resin-monomers. Dent Mater 2006; 22: 785-791.

29) Park YJ, Chae KH, Rawls HR. Development of a new photoinitiation system for dental light-cure composite resins. Dent Mater 1999; 15: 120-127.

30) Jandt KD, Mills RW, Blackwell GB, Ashworth SH. Depth of cure and compressive strength of dental composites cured with blue light emitting diodes (LEDs). Dent Mater 2000; 16: $41-47$

31) Teshima W, Nomura Y, Tanaka N, Urabe H, Okazaki M, Nahara Y. ESR study of camphorquinone/amine photoinitiator systems using blue light-emitting diodes. Biomaterials 2003; 24: 2097-2103.

32) Teshima W, Nomura Y, Tanaka N, Shibata S, Shintani T, Shirai K, Urabe H, Nahara Y, Shintani H. Kinetic analysis of photopolymerized dental resins supplemented with new photoinitiators cured by violet LEDs. J J Concerve Dent 2004; 47: 442-453.

33) Masuhara E, Komiya S, Sawamoto T, Satou Y. A photoinitiator composition for visible light polymerizable adhesives. Japanese Patent No. 3442776 (2003).

34) Ikemura K, Ichizawa K. Visible-light polymerizable composition. Japanese Patent Toku-Kai 2005-171213; 2005.

35) Moriyama S, Murata S, Tanaka M, Makino Y. A phosphine oxide compound. Japanese Patent Open, Toku-kai 2005225793; 2005.

36) Imai Y, Kadoma Y, Kojima K. A photoinitiator. Japanese Patent Toku-Kai-Hei 3-243602 (1991).

37) Bhattacharya AK, Thyagarajan G. The Michaelis-Arbuzov rearrangement. Chem Rev 1981; 81: 415-430.

38) Murphy PJ. Practical approach in chemistry Organophosphorus reagents, 1st ed. New York: Oxford University Press Inc; 2004. p.172-185.

39) Ikemura K, Ichizawa K, Jyougetsu Y. Camphorquinone derivative having acylphosphine oxide group, and containing the same, photopolymerization catalyst and photo/chemical polymerization catalyst, and containing these, hardenable 
composition. International Patent Application No. PCT/ JP2006/304911, WO/2007/105296; 2006.

40) De Groot JH, Dillingham K, Deuring H, Haitjema HJ, van Beijima FJ, Hodd K, Norrby S. Hydrophilic polymeric acylphosphine oxide photoinitiators/crosslinkers for in vivo blue-light photopolymerization. Biomacromol 2001; 2: 12711278.

41) Lai JH, Johnson AE. Measuring polymerization shrinkage of photo-activated restorative materials by a water-filled dilatometer. Dent Mater 1993; 16: 172-176.

42) Davis MJ, Gawne G, Green PN, Green WA. The synthesis and properties of a novel series of water soluble thioxanthone photoinitiators. Spec Chem 1986; 6: 363-369.

43) Pedersen CJ. Cyclic polyethers and their complexes with metal salts. J Am Chem Soc 1967; 89: 7017-7036.

44) Ikemura K, Ichizawa K, Fuchigami K. A curable composition comprising clathrate compounds. Japanese Patent Open No. Toku-Kai-2005-248137 (2005).

45) Ulman A. An introduction to ultrathin organic films: From Langmuir-Blodgett to self-assembly. San Diego: Academic Press Inc; 1991.

46) Hendrixson RR, Mack MP, Palmer RA, Ottolenghi A, Ghirardelli RG. Oral toxicity of the cyclic polyethers - 12crown-4, 15-crown-5, and 18-crown-6 in mice. Toxicol Appl Oharmacol 1978; 44: 263-268.

47) Tay FR, Pashley DH. Permeability of single-step, self-etch adhesives - the cost of saving time. "Self-etching Primer: Current Status and Its Evolution". Proceedings of the International Symposium '01 in Tokyo, First Edition, 2003. p. 23-39.

48) De Munck J, Arita A, Shirai K, Van Landuyt KL, Coutinho E, Poitevin A, Peumans M, Lambrechts P, Van Meerbeek B. Microrotary fatigue resistance of a HEMA-free all-in-one adhesive bonded to dentin. J Adhes Dent 2007; 9: 373-379.

49) Nishiyama N, Suzuki K, Yoshida H, Teshima H, Nemoto K. Hydrolytic stability of methacrylamide in acidic aqueous solution. Biomaterials 2004; 25: 965-969.

50) Katsuno K, Manabe A, Itoh K, Nakamura Y, Wakumoto S, Hisamitsu H, Yoshida T. Contact dermatitis caused by 2 HEMA and GM dentin primer solutions applied to guinea pigs and humans. Dent Mater J 1996; 15: 22-30.

51) Ichizawa K, Ikemura K. A pH-controlled adhesive composition. Japanese Patent Open No. Toku-Kai-200731339 (2007).

52) Freedman G. First impressions. Dentistry Today 2009; 28: 1.

53) Takeuchi F, Mizuta Y. Photocurable resin composition containing anthraquinone derivative. International Patent WO 2007/074782 A1 (2007).

54) Angiolini L, Caretti D, Carlini C. Polymeric photoinitiators bearing side-chain benzoyldiphenylphosphine oxide moieties for UV curable coatings. J Appl Polym Sci 1994; 51: 133143.

55) Angiolini L, Caretti D, Carlini C. Polymeric systems bearing side-chain 2,6-dimethylbenzoyl-diphenylphosphine oxide moieties for UV curable coatings: Synthesis and photoinitiation activity. J Appl Polym Sci 1995; 57: 519531. 